MIT-CTP-2826

hep-th/9902118

\title{
Marginal Deformations from Branes
}

\author{
Joshua Erlich, Amihay Hanany and Asad Naqvi \\ Center for Theoretical Physics, \\ Massachusetts Institute of Technology, Cambridge, MA 02139, USA \\ jerlich@ctp.mit.edu, hanany@ctp.mit.edu, naqvi@ctp.mit.edu
}

\begin{abstract}
We study brane configurations for four dimensional $\mathcal{N}=1$ supersymmetric gauge theories with quartic superpotentials which flow in the infrared to manifolds of interacting superconformal fixed points. We enumerate finite $\mathcal{N}=2$ theories, from which a large class of marginal $\mathcal{N}=1$ theories descend. We give the brane descriptions of these theories in Type IIA and Type IIB string theory. The Type IIB descriptions are in terms of D3 branes in orientifold and generalized conifold backgrounds. We calculate the Weyl and Euler anomalies in these theories, and find that they are equal in elliptic models and unequal in a large class of finite $\mathcal{N}=2$ and marginal $\mathcal{N}=1$ non-elliptic theories.
\end{abstract}




\section{Introduction}

Finite theories in four dimensions have a rich structure, much of which has been a subject of recent interest. Finite gauge theories having vanishing $\beta$ functions and anomalous dimensions are conformal, and without divergences when a perturbative expansion is valid. Such theories contain dimensionless parameters which are independent of scale and couple to marginal operators. Finite theories often belong to a continuous manifold of scale invariant theories. The flow along these theories is characterized by a number of marginal operators equal to the dimension of the manifold of fixed points. Those marginal operators which when added to the action of a conformal theory generate a flow along the manifold of fixed points are called exactly marginal operators [1]. Certain four dimensional field theories, although having a manifold of conformal fixed points, do not have the property that all anomalous dimensions are zero. These theories are not finite by the definition above, but in the infrared they contain some number of exactly marginal operators.

One way of generating theories with marginal deformations is to start with a theory with negative $\beta$ functions, and perturb it by an irrelevant operator in the ultraviolet. In some cases, upon flowing to the infrared the irrelevant deformation develops a negative anomalous dimension in such a way that it becomes marginal.

We study $\mathcal{N}=1$ supersymmetric gauge theories with quartic superpotentials and marginal deformations in the infrared. A large class of such theories are obtained from finite $\mathcal{N}=2$ theories by integrating out the adjoint chiral multiplet [1, 2]. We give brane descriptions for these theories and identify the exactly marginal operators with motions of NS5 branes in Type IIA configurations, or with NS two-form fields in the Type IIB picture. We enumerate all finite $\mathcal{N}=2$ theories with one and two factors of classical gauge groups, and discuss generalizations.

In Section 2 we discuss, from a field theoretic point of view, the conditions for existence of exactly marginal operators. In Section 3 we review configurations of intersecting NS5 branes and D4 branes in the presence of D6 branes and O6 plane backgrounds in Type IIA string theory. We suggest a simple criterion which when imposed on the brane configurations, gives field theories with exactly marginal operators. For a certain class of brane configurations (the elliptic models) in Type IIA string theory, we give a T-dual picture in Type IIB. We generalize the results of [3, 4, 5] to more complicated conical singularities, and discuss orientifolds in $\mathcal{N}=1$ and $\mathcal{N}=2$ theories, generalizing the analysis in [6]. In Section $\mathbb{t}$ we list all conformal

$\mathcal{N}=2$ theories with one or two classical gauge groups and give the corresponding brane descriptions. In Section 5 we present a more general analysis of supergravity descriptions, and argue that a large class of non-elliptic models which have a Type IIA description can 
not satisfy the supergravity condition relating the Euler and Weyl anomalies of theories with supergravity descriptions [7], while all elliptic models considered are shown to satisfy that relation.

\section{$2 \mathcal{N}=1$ theories with $Q^{4}$ type superpotentials}

Following the discussion of Leigh and Strassler [1] we argue that four-dimensional $\mathcal{N}=2$ supersymmetric theories with vanishing one loop $\beta$ function have associated with them $\mathcal{N}=$ 1 supersymmetric theories which have a manifold of fixed points. These $\mathcal{N}=1$ theories are obtained from the $\mathcal{N}=2$ finite theories by integrating out the adjoint chiral multiplet, giving rise to a quartic superpotential in the low energy theory. The theory with quartic superpotential has a line of fixed points: In the space of coupling parameters of the theory, there is a line along which the theory is conformally invariant. At each fixed point, the Lagrangian of the theory can be deformed in such a way that the theory remains conformally invariant. The operator which can be added to the Lagrangian to deform the theory in this way is said to be an exactly marginal operator.

The exact NSVZ $\beta_{g}$ function [8] for the gauge coupling in a supersymmetric gauge theory with chiral superfields $\phi_{i}$ in representations $r_{i}$ with Dynkin indices $T\left(r_{i}\right)$ and anomalous dimensions $\gamma_{i}$ (the normalization for the anomolous dimensions here is a factor of two larger than in some other references), and adjoint Dynkin index $G$, is proportional to

$$
\beta_{g} \propto 3 G-\sum_{i} T\left(r_{i}\right)\left[1-\gamma_{i}\right]
$$

The couplings in the superpotential, which are schematically of the form $W=h \phi_{1} \phi_{2} \cdots \phi_{k}$ are renormalized according to a similar equation for their $\beta_{h}$ functions given by

$$
\beta_{h} \propto \sum_{i}\left(d_{i}+\frac{1}{2} \gamma_{i}\right)-3
$$

where $d_{i}$ is the canonical dimension of the field $\phi_{i}$. The theory is at a fixed point when all of the $\beta$ functions in the theory vanish. The anomalous dimensions are functions of the couplings, so each equation $\beta_{g}=0$ or $\beta_{h}=0$ provides one relation between the couplings. Generically there will be isolated solutions to these equations, or none at all, but if the equations for the $\beta$ functions in terms of the $\gamma_{i}$ are linearly dependent then there may be a manifold of fixed points of codimension equal to the rank of the set of linearly independent $\beta$ functions. In that case there are marginal operators along the manifold of fixed points 
which deform the infrared theory from one fixed point to another.

For $\mathcal{N}=2$ theories with massless hypermultiplets $Q_{I}$ in representations $r_{I}$ of the gauge group, vanishing of the one loop $\beta_{g}$ function is sufficient for the theory to be exactly finite. For any value of the gauge coupling $g$, the theory is conformally invariant and so has a line of fixed points generated by the $\operatorname{Tr}\left(F^{2}\right)$ operator. The anomalous dimensions of all the fields vanish along this line and the theory is finite.

We can now give mass to the adjoint chiral multiplet. With $\mathcal{N}=1$ chiral multiplets $Q^{I}, \tilde{Q}^{I}$ and adjoint chiral multiplet $\Phi$, the superpotential is of the form $W=h \sum_{I} \operatorname{Tr} Q^{I} \Phi \tilde{Q}^{I}+$ $m \operatorname{Tr} \Phi^{2}$, where the trace is over the gauge indices, and $h$ is proportional to the gauge coupling $g$. Integrating out the adjoint gives

$$
W=-\frac{h^{2}}{4 m}\left(\sum_{I} \operatorname{Tr} Q^{I} \tilde{Q}^{I}\right)^{2} .
$$

Depending on the structure of the adjoint $\Phi$ it may be necessary to add Lagrange multipliers to impose constraints on $\Phi$, e.g. tracelessness. In that case the exact form of $W$ will change, but the $Q^{4}$ structure will remain the same, which is the important aspect for our purposes. The $Q^{4}$ coupling will flow to some value $\tilde{h}$ in the far infrared that will depend on the dynamical scale and adjoint mass. We can relax the condition that the contribution from each term in the superpotential have the same coupling (assuming no flavor symmetry), and consider the possibility that a fixed point with all of the couplings equal belong to a continuous family of conformal theories with $W=\sum_{I<J} h_{I J}\left(\operatorname{Tr} Q^{I} \tilde{Q}^{I}\right)\left(\operatorname{Tr} Q^{J} \tilde{Q}^{J}\right)$. If the parent $\mathcal{N}=2$ theory has vanishing one loop $\beta_{g}$ function, then the $\beta$ functions of the corresponding $\mathcal{N}=1$ theory are, according to (1) and (2),

$$
\begin{aligned}
\beta_{g} & =G+\sum_{I} T\left(r_{I}\right) \gamma_{I} \\
\beta_{h_{I J}} & =1+\gamma_{I}+\gamma_{J} .
\end{aligned}
$$

At a fixed point, $\beta_{g}=\beta_{h_{I J}}=0$. These are $n(n+1) / 2+1$ linear equations for the $n a$ priori unrelated anomalous dimensions. The diagonal components $I=J$ of (4) completely determine the value of the $n$ anomalous dimensions. The solutions are $\gamma_{I}=-\frac{1}{2}$, and the vanishing of $\beta_{g}$ then follows from the vanishing of the one loop beta function of the parent $\mathcal{N}=2$ theory. Hence, the condition that $\beta_{g}$ vanish is redundant and we find a line of fixed points. In fact, each of the off diagonal $\beta_{h_{I J}}=0$ equations is linearly dependent on the diagonal equations, so if there are $n$ fields $Q^{I}$ then there is a $n(n-1) / 2+1$ dimensional manifold of fixed points. The fixed points of the theory with superpotential (3) lie on a one 
dimensional submanifold. There is a linear combination of the gauge coupling $W_{\alpha} W^{\alpha}$ and the superpotential $W$ which varies over the line of fixed points and deforms the theory along it.

The simplest example of this phenomenon is obtained from $\mathcal{N}=2 \mathrm{SU}(N)$ Yang-Mills theory with $N_{f}$ hypermultiplets $Q^{I}$ in the fundamental representation of the gauge group. Upon integrating out the adjoint, taking into account the tracelessness condition, the effective superpotential is

$$
W \sim Q_{\alpha}^{I} \tilde{Q}_{J}^{\alpha} Q_{\beta}^{J} \tilde{Q}_{I}^{\beta}-\frac{1}{N_{c}} Q_{\alpha}^{I} \tilde{Q}_{I}^{\alpha} Q_{\beta}^{J} \tilde{Q}_{J}^{\beta}
$$

This theory was studied in [1] in relation to $\mathcal{N}=1$ duality.

In the following we review brane configurations involving intersecting NS5 branes and D4 branes in Type IIA string theory for these types of models and motivate a relation between marginal deformations and NS5 brane motion. We enumerate theories with a small number of gauge group factors which behave similarly to the theories discussed above and give their corresponding brane constructions in Type IIA and Type IIB string theory. We will also see that only a large class of these types of theories have unequal Weyl and Euler anomalies,

and following [0, 9] therefore do not have useful supergravity descriptions in the sense of Maldacena's conjecture [10].

\section{Brane configurations in Type II string theories}

In this section we identify marginal operators with deformations of brane configurations or geometry in Type II string theories. We suggest that translations of straight NS5 branes in Type IIA configurations correspond in the field theory to motion along a manifold of fixed points in the infrared. Much work has been done on understanding non-perturbative effects in field theories from string theory. The three methods which have been employed for this purpose rely on: (a) string theory in a non-trivial geometry; (b) the world volume theory of branes probing a non-trivial (typically singular) geometry; and (c) the world volume theory of branes in a brane configuration in a flat background. These different descriptions of the field theories can often be related by various dualities. In the following we review configurations of intersecting branes in Type IIA string theory in flat Minkowski space for the theories alluded to in the previous section, and then discuss the T-duality which maps these configurations to D-branes probing singular geometries in Type IIB string theory. 


\section{Type IIA brane constructions and marginal deformations}

Configurations of intersecting D4 branes and NS5 branes in Type IIA string theory in flat Minkowski space are well studied (for a review see [11). In this paper, we will be using NS5, NS5', D4 and D6 branes in the following directions:

\begin{tabular}{|c|c|c|c|c|c|c|c|c|c|c|}
\hline & 0 & 1 & 2 & 3 & 4 & 5 & 6 & 7 & 8 & 9 \\
\hline NS5 & + & + & + & + & + & + & & & & \\
\hline NS5' & + & + & + & + & & & & & & + \\
\hline D4 & + & + & + & + & & & {$[-]$} & & & \\
\hline D6 & + & + & + & + & & & & + & + & + \\
\hline
\end{tabular}

The configuration shown in Fig. [ 10 consists of NS5, D4 and D6 branes.

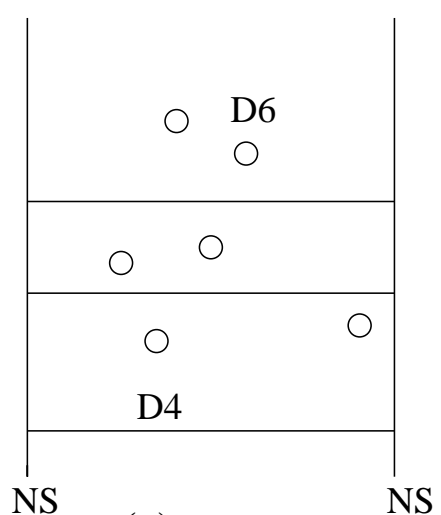

(a)

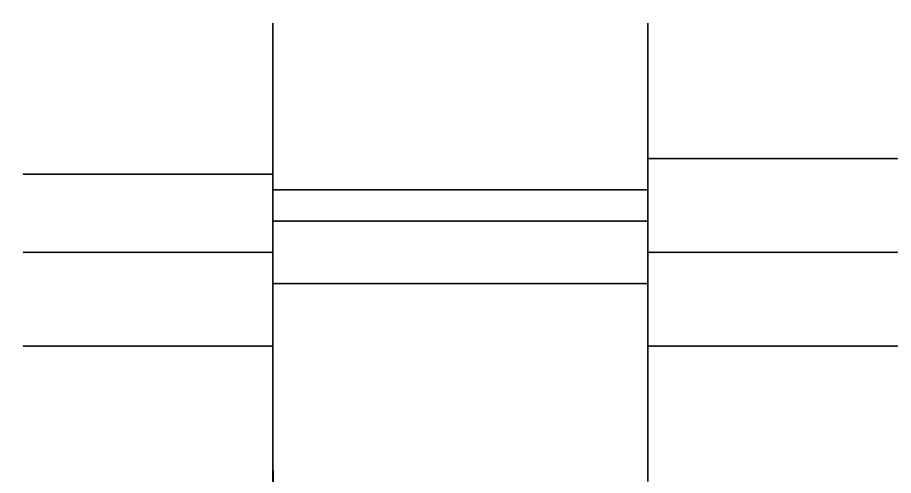

(b)

Figure 1: $\mathrm{SU}(N)$ with $2 N \square$ hypermultiplets.

The D4 branes end on NS5 branes and are of finite extent $L$ in the $x_{6}$ direction. The NS5 and D4 branes are at a point in $x_{7}, x_{8}, x_{9}$. If there are $N$ D4 branes and $N_{f}$ D6 branes, the field theory on the world volume of the D4 branes at length scales much larger than $L$ is a four dimensional $\mathcal{N}=2 \mathrm{SU}(N)$ gauge theory with $N_{f}$ hypermultiplets in the fundamental representation (corresponding to the strings ending on the D6 branes and D4 branes). Instead of using D6 branes, flavors can be added with $N_{f}$ semi-infinite D4 branes oriented in the same direction as the other D4 branes. The two ways of adding flavors to the gauge theory can be related by passing the D6 branes through the NS5 branes, at which point a D4 brane is created stretching between the D6 and the NS5 branes [12]. 
As discussed in [13], the force exerted by the D4 branes on NS5 branes causes the NS5 branes to bend in the $x_{6}$ direction. The distance between the NS5 branes corresponds to the gauge coupling: $1 / g^{2}=L / \lambda$ where $\lambda$ is the string coupling constant. The running of the gauge coupling is nicely reflected by the bending of the NS5 branes. If the beta function of the gauge theory is zero, the NS5 branes can be at fixed values of $x_{6}$ with no bending asymptotically. This will be the case when $N_{f}=2 N$, with $N$ semi-infinite D4 branes from the left and $N$ from the right of each NS5 brane (if there are no D6 branes). In the presence of D6 branes, the requirement for there to be no relative bending of the NS5 branes asymptotically is that the linking number of each NS5 brane is identical: If $L_{4}^{i}, R_{4}^{i}$ are the number of D4 branes attached to the left and right of the $i$ th NS5 brane and $l_{6}^{i}, r_{6}^{i}$ are the number of D6 branes to the left and right of that NS5 brane, then the linking number $\left(L_{4}^{i}-R_{4}^{i}\right)+\frac{1}{2}\left(r_{6}^{i}-l_{6}^{i}\right)$ must be the same for each NS5 brane. (O6 $6^{ \pm}$planes, if present, would contribute to linking numbers as if they were $( \pm)$ a pair of physical D6 branes. O4 planes passing through an NS5 brane change sign, and contribute $( \pm 2)$ units of D4 brane charge to the linking number of the NS5 brane through which they pass.) If a configuration has equal nonzero linking numbers for each NS5 brane, then sixbranes can be added past the leftmost or rightmost NS5 brane such that all linking numbers will vanish. The additional sixbranes do not affect the field theory on the fourbranes since they can be moved to infinity.

If one of the NS5 branes is rotated out of the $\left(x_{4}, x_{5}\right)$ plane and into the $\left(x_{7}, x_{8}\right)$ plane, then the D4 branes can no longer slide along the NS5 branes and the Coulomb branch of the theory is lifted. This corresponds to giving a mass to the adjoint proportional to $\tan \theta$ where $\theta$ is the angle of rotation of the NS5 branes. (This dependence should only be trusted for masses well below the string scale; otherwise the adjoint might decouple at a scale higher than the string scale, but we are ignoring all string states.) The field theory living on the world-volume of the $\mathrm{D} 4$ branes can be obtained by integrating out the adjoint. We get a $\mathcal{N}=1$ theory with a quartic superpotential. If we start from a theory which has no asymptotic bending of the NS5 branes, i.e. $N_{f}=2 N$, it is easy to see that there is an exactly marginal operator which generates a manifold of fixed points (as discussed in detail in the previous section).

An obvious extension of the brane configuration discussed above is shown in Fig. 2 . There are $M+1$ NS5 branes labeled by $\alpha=1, \ldots, M+1$ with $k_{\alpha}$ D4 branes stretched between the $\alpha$ th and $(\alpha+1)$ th NS5 branes, and $d_{\alpha}$ D6 branes at points between the $\alpha$ th and $(\alpha+1)$ th NS5 brane. The gauge group of the four-dimensional theory is $\prod_{\alpha=1}^{M} \mathrm{SU}\left(k_{\alpha}\right)$. The matter content contains $d_{\alpha}$ hypermultiplets in the fundamental representation of $\mathrm{SU}\left(k_{\alpha}\right)$ (except for $\mathrm{SU}\left(k_{1}\right)$ and $\mathrm{SU}\left(k_{M}\right)$ which have $d_{1}+k_{0}$ and $d_{M}+k_{M+1}$ such hypermultiplets) and bifundamental hypermultiplets transforming as $\left(k_{1}, \bar{k}_{2}\right) \oplus\left(k_{2}, \bar{k}_{3}\right) \oplus \ldots\left(k_{M-1}, \bar{k}_{M}\right)$. The beta 


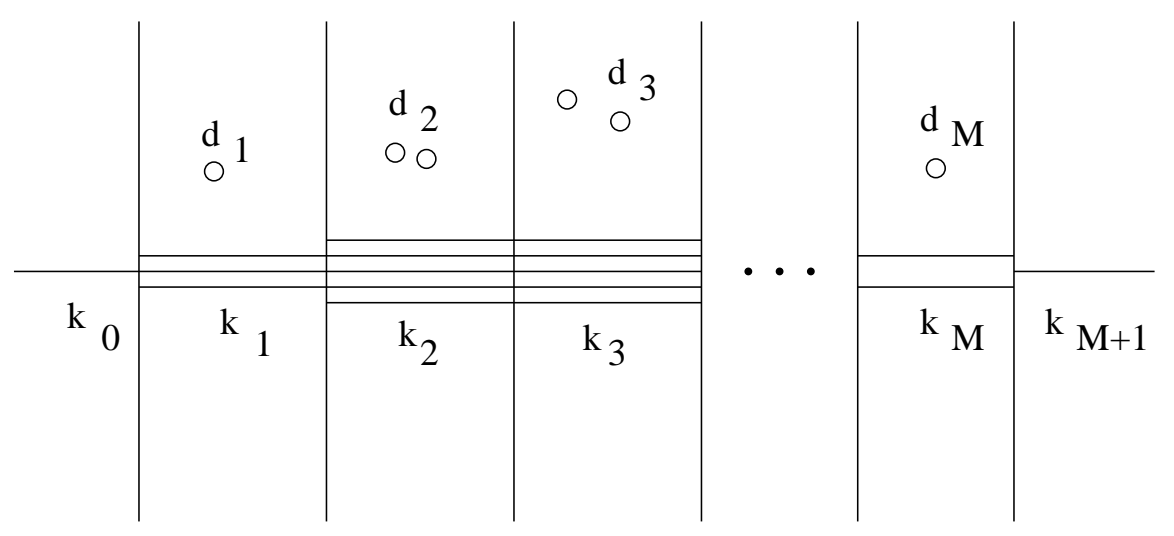

Figure 2: The brane configuration corresponding to the $\mathrm{SU}\left(k_{1}\right) \times \mathrm{SU}\left(k_{2}\right) \times \cdots \times \mathrm{SU}\left(k_{M}\right)$ theory with bifundamentals and $k_{0}$ flavors of $\mathrm{SU}\left(k_{1}\right)$ and $k_{M+1}$ flavors of $\mathrm{SU}\left(k_{M}\right)$. The vertical lines represent NS5 branes, the horizontal lines are D4 branes and the circles are D6 branes orthogonal to the $\left(x_{4}, x_{6}\right)$ plane drawn here..

function for $\mathrm{SU}\left(k_{\alpha}\right)$ is

$$
b_{0, \alpha}=-\left(2 k_{\alpha}-k_{\alpha-1}-k_{\alpha+1}-d_{\alpha}\right) .
$$

Rotating an NS5 brane into the $\left(x_{7}, x_{8}\right)$ plane gives a mass to the adjoint chiral multiplets of the gauge groups to the left and right of that NS5 brane. The contribution to the masses of the adjoints is of opposite sign to the left and right of the rotated NS5 brane. This breaks $\mathcal{N}=2$ to $\mathcal{N}=1$ and gives rise to a quartic superpotential for those fields which transform under the gauge groups to the left and right of the NS5 brane as discussed in Section 2 .

The existence of a marginal deformation in the infrared is motivated by the absence of logarithmic bending of the NS5 branes in configurations with vanishing linking numbers, and hence the absence of a scale in those theories. The independent translations of the NS5 branes are intuitively expected to correspond to marginal deformations in the infrared precisely because they are deformations not associated with a dimensionful parameter. Rotations of NS5 branes induce masses for the adjoint fields, and are thus relevant deformations in the ultraviolet. These relevant deformations also induce a flow along the manifold of fixed points in the infrared theory, but do not correspond to exactly marginal operators. More precisely, there is a linear combination of the gauge coupling, $W^{\alpha} W_{\alpha}$, and the couplings in the infrared superpotential, $W$, which when added to the action of the infrared theory deforms the theory along the line of fixed points. At each fixed point there are independent marginal operators corresponding to the gauge coupling and the terms in the superpotential (corresponding to motions of the NS5 branes), but by adding only one combination of marginal operators to the action does the theory remain conformally invariant. 
The proposed intuition relating deformations of brane configurations with straight branes and exactly marginal operators is generally absent in a curved spacetime background because of the scale induced by the curvature. For example, in the presence of D6 branes and orientifolds, the intuition relating NS5 brane motion to marginal operators is more tenuous, and we identify marginal deformations only if the linking numbers of all NS5 branes, including the contributions due to the orientifold charges, vanish. In the $\mathcal{N}=2$ case, translations of NS5 branes correspond to variation of the gauge coupling, which is indeed an exactly marginal deformation for the finite theories. In the $\mathcal{N}=1$ case, with rotated NS5 branes, the relation between translations of NS5 branes and exactly marginal operators is not as immediately evident, but we will see that theories with straight rotated branes generally have marginal deformations as well. The identification of the exactly marginal operator corresponding to the NS5 brane motion can be made at weak coupling (where it corresponds to changing the gauge coupling - the exactly marginal operator is $\operatorname{Tr} F^{2}$ ). At strong coupling, the operator corresponding to the NS5 brane motion cannot be identified easily. However, the existence of such an operator can be seen from the brane picture.

\section{Elliptic Models}

An interesting set of models is obtained if the $x_{6}$ direction is compactified on a circle of length $L$, with D6 branes between any pair of NS5 branes. Then $k_{0}=k_{M+1}$ by definition, and the gauge group is $\prod_{\alpha=0}^{M} \mathrm{SU}\left(k_{\alpha}\right) \times \mathrm{U}(1)$. The extra $\mathrm{U}(1)$ is from uniform translation of the fourbranes along the $\left(x_{4}, x_{5}\right)$ directions. The hypermultiplet spectrum is as before except that the $k_{0}$ and $k_{M+1}$ fundamental hypermultiplets before compactification combine to give a bifundamental transforming under $\mathrm{SU}\left(k_{M}\right)$ and $\mathrm{SU}\left(k_{0}\right)$ and another bifundamental hypermultiplet transforming under $\mathrm{SU}\left(k_{0}\right)$ and $\mathrm{SU}\left(k_{1}\right)$. None of the hypermultiplets is charged under the extra $U(1)$, so the $U(1)$ factor decouples from the theory and we will not be interested in it. The beta function for each group is still given by (6). It is easy to see that the only way to get zero or negative beta functions for all groups is to set $d_{\alpha}=0$ and $k_{\alpha}=N$ for all $\alpha$. This implies that all $b_{0, \alpha}=0$, and there is no relative bending of the NS5 branes.

It is worthwhile to note that the relation between bending of NS5 branes and the beta function must be reconsidered in elliptic models. The linking numbers are not well defined $a$ priori because the notion of left and right is imprecise on the circle. However, the relation can be made more precise by introducing a fundamental domain on the circle. If the circle is cut at any point and treated as a theory on a non-compact background, then the linking numbers on any NS5 brane must be equal for the theory to be finite. Choosing a different fundamental domain, i.e. cutting the circle at a different location, will lead to different linking numbers 
in general, but the equality of linking numbers is unaffected by this choice. Furthermore, the beta functions are related to relative differences between linking numbers of neighboring NS5 branes just as in the non-compact case. On the circle, marginal deformations are related to both translations of NS5 branes along the circle and variations of the radius of the compact direction.

\section{Type IIB descriptions}

For the elliptic models we can perform a T-duality along the compact $x_{6}$ direction. A configuration with a set of $N_{5}$ parallel NS5 branes in Type IIA string theory is mapped to Type IIB on $\mathrm{A}_{N_{5}-1} \times R^{6}$, where $\mathrm{A}_{N_{5}-1}$ is a $\mathbb{C}^{2} / \mathbb{Z}_{N_{5}}$ orbifold type ALE space. The $N \mathrm{D} 4$ branes which wrap the compact direction are mapped to D3 branes. So the configuration discussed above with a compact $x_{6}$ direction is T-dual to $N$ D3 branes at an $\mathrm{A}_{N_{5}-1}$ singularity. The D3 branes occupy the $\left(x_{0}, x_{1}, x_{2}, x_{3}\right)$ directions and the ALE space is in the $\left(x_{6}, x_{7}, x_{8}, x_{9}\right)$ directions.

Turning on two-form B-fields which have non-vanishing flux over the vanishing two-cycles at the singularity corresponds to moving the NS5 branes in the $x_{6}$ direction. In the field theory this corresponds to variations in the gauge couplings [14. There is a question of the ordering of the NS5 branes which we will not discuss here. The formalism for studying D branes at orbifold singularities was developed in [14]. The world volume theory on the D3 branes is determined by the orbifold action on spacetime and Chan-Paton factors (and can be encoded in quiver diagrams or generalizations such as in [14, 15, 16, 17]), and is the same as the theory that we started with in the Type IIA picture before performing the T-duality.

The $\mathcal{N}=1$ theories correspond to brane configurations with rotated NS5 branes. On the Type IIB side, the resolved singular space varies over the $\left(x_{4}, x_{5}, x_{6}, x_{7}, x_{8}, x_{9}\right)$ directions, and can be described by a type of blowup of the orbifold singularity, as discussed in [3, 4, 5, 18] for the case of the $A_{1}$ type singularity. Alternatively, as described in [19], the space can be thought of as a complex deformation of the orbifold singularity, re-embedded in a weighted projective space. In the complex deformation approach, the algebraic form of the orbifold singularity is changed so as to smooth out the singularity. The orbifold singularity is invariant under a $\mathbb{C}^{*}$ action which is absent in the deformed curve. By re-embedding the deformed curve in a weighted projective space we restore the $\mathbb{C}^{*}$ action, and the resulting curve is the generalized conifold. More explicitly, the $\mathrm{A}_{k}$ orbifold singularity is given by a curve of the form

$$
x^{k+1}+y^{2}+z^{2}=0 .
$$

It is invariant under a $\mathbb{C}^{*}$ action with weights $\left(1, \frac{k+1}{2}, \frac{k+1}{2}\right)$ for $(x, y, z)$. 
The multiplicity of the singularity is $k$, equal to the rank of the corresponding $\mathrm{A}_{k}$ gauge group, and the singularity can be thought of as a bouquet of $k$ spheres shrinking to zero size. A general deformation of the curve must have at least $k$ parameters specifying the deformation. A deformation is called miniversal if it is specified by a number of parameters equal to the multiplicity $k$ of the singularity, and if any deformation is equivalent to that deformation. One can generate a miniversal deformation of a singular curve by adding all polynomials to the function specifying the curve, modulo polynomials times the first partial derivatives of the non-deformed curve [20]. For example, the complex deformation of the curve (7) is of the form 19, 21]

$$
x^{k+1}+\sum_{m=1}^{k} t_{m} x^{k-m}+y^{2}+z^{2}=0 .
$$

This deformed curve is then projectivized by introducing a new variable $s$ with projective weight 1 , giving the $\mathrm{A}_{k}$ generalized conifold,

$$
x^{k+1}+s^{k+1} \sum_{m=1}^{k} t_{m}\left(\frac{x}{s}\right)^{k-m}+y^{2}+z^{2}=0 .
$$

We can replace the deformation parameters $t_{i}$ by angles $\theta_{i}$, and rewrite (9),

$$
y^{2}+z^{2}=\prod_{m=1}^{k+1}\left(s \cos \theta_{m}+x \sin \theta_{m}\right) .
$$

In this form, we identify the angles $\theta_{i}$ with the rotation angles of the NS5 branes in the Type IIA picture. The masses of the adjoints are given by the relative angles, $m_{i} \propto \tan \left(\theta_{i+1}-\theta_{i}\right)$, at least for small relative angles $\theta_{i+1}-\theta_{i}$, and satisfy the periodicity condition $\sum_{i} m_{i}=0$. For example, the $\mathrm{A}_{1}$ curve, corresponding to a pair of NS5 branes on a circle, interpolates between the $\mathcal{N}=2$ orbifold $\left(\theta_{1}=0, \theta_{2}=0\right)$ and the $\mathcal{N}=1$ conifold $\left(\theta_{1}=0, \theta_{2}=\pi / 2\right)$. The generalized conifold (9) was also shown in [19] to correspond to the moduli space of the Higgs branch of the corresponding $\mathcal{N}=1$ gauge theory by studying solutions to the $D$ - and $F$-flatness equations.

\section{Adding Orientifolds}

We can obtain a rich class of theories by adding orientifolds to the Type IIA brane configurations described above. In fact, as we will see in the next section, the finite $\mathcal{N}=2$ theories with simple classical groups and products of two factors, and with at most two-index matter, 
can be obtained in the Type IIA brane picture if we use orientifold planes. We will mostly be interested in O6 planes occupying the $\left(x_{0}, x_{1}, x_{2}, x_{3}, x_{7}, x_{8}, x_{9}\right)$ directions (parallel to D6 branes introduced earlier) and $\mathrm{O} 4$ planes parallel to the D4 branes.

Consider a collection of NS5 branes (and their images under orientifold reflection) at some distance from an O6 plane. D4 branes can stretch between pairs of NS5 branes, but only in configurations symmetric under the orientifold reflection. For example, the configuration with $2 N$ D4 branes ( $N$ physical D4 branes and their images) stretched between a NS5 brane and its orientifold image, as discussed in [22], corresponds to an $\mathcal{N}=2 \mathrm{SO}(2 N)$ or $\operatorname{USp}(2 N)$ theory, depending on the sign of the orientifold charge. We can add $N_{f}$ D6 branes and their images to the above setup. Then we get $2 N_{f}$ fundamental chiral multiplets under the gauge group, or $N_{f}$ hypermultiplets. If the sixbrane $\mathrm{RR}$ charge of the $\mathrm{O}^{ \pm}$plane is $+4(-$ 4) (physical charge $+2,(-2))$, we get an $\operatorname{SO}(2 N)(\operatorname{USp}(2 N))$ gauge theory with $\operatorname{USp}\left(2 N_{f}\right)$ $\left(\mathrm{SO}\left(2 N_{f}\right)\right)$ flavor symmetry. As before, this way of adding flavors is equivalent to adding semi-infinite D4 branes. The absence of bending of the NS5 branes requires vanishing beta function in the gauge theory. Hence we need $N_{f}=2 N-2$ for the case of $\mathrm{O}^{+}$and $N_{f}=2 N+2$ for the case of $\mathrm{O}^{-}$. This can be understood on the basis of the fourbrane charge induced on the NS5 branes by the presence of the O6 plane. Since the orientifold carries sixbrane charge, it interacts with the NS5 brane as though there were sixbranes or "anti-sixbranes" present. That a fourbrane charge is induced is evident by conservation of RR charge and the fact that pulling a sixbrane through a NS5 brane produces a fourbrane connecting them [12]. Then in order to balance the force of the fourbrane charges on the NS5 brane, the number of D4 branes attached to the left and right sides of each NS5 must differ. In other words, the linking number of each NS5 brane should be the same.

In the presence of an O6 plane as above, NS5 branes not stuck to the O6 plane can be rotated out of the $\left(x_{4}, x_{5}\right)$ plane into the $\left(x_{7}, x_{8}\right)$ plane. Each NS5 brane and its image are rotated in opposite directions because the configuration has to remain symmetric under reflection about the orientifold plane, $\left(x_{4}, x_{5}, x_{6}\right) \rightarrow\left(-x_{4},-x_{5},-x_{6}\right)$. As a result of this rotation, the D4 branes are fixed at the origin and cannot slide between the NS5 branes anymore. As usual, this corresponds in the field theory to giving a mass to the adjoint chiral multiplet, breaking $\mathcal{N}=2$ to $\mathcal{N}=1$ and lifting the Coulomb branch of the theory. The field theory analysis shows that by integrating out the adjoint, at low energies, we will see a marginal quartic superpotential in the flavor superfields.

An interesting $\mathcal{N}=1$ configuration is obtained when the angle of rotation of each NS5 brane is $\pi / 2$. In that case, the two NS5 branes become parallel to each other and to the O6 plane and the D4 branes can slide off between the two NS5 branes. This shows that there must be a field in the field theory which is becoming massless at this point. The additional 
field was shown in [23, 24] to tranform as a symmetric tensor under the $\mathrm{SO}(2 N)$ gauge group or antisymmetric tensor under the $\operatorname{USp}(2 N)$ gauge group; hence, at this point the adjoint chiral multiplet is substituted by the opposite type of two index tensor. The conjecture that NS5 brane motion corresponds to exactly marginal deformations leads to a prediction that the theory on the D4 branes of this brane configuration has a manifold of fixed points, but only for the USp case. The SO theory comes from an $\mathrm{O}^{+}$plane, whose sixbrane charge parallel to the NS5 branes cannot be canceled. This theory will be discussed further in Section 4 . Even though we expect there to be marginal deformations of the USp theory, we cannot conclude that the manifold of fixed points of this theory is related to the manifold of fixed points of the theory without the symmetric tensor. The interchange of the adjoint and tensor fields involves passing through mass scales for these fields larger than the string scale. In order to trace what happens to these theories we would have to include the effects of string states which we have been ignoring. Furthermore, since rotations of NS5 branes correspond to relevant deformations, we should not expect in general to remain on the same manifold of fixed points after such perturbations of the theory. Translations of NS5 branes are expected to yield exactly marginal deformations of the theory. It is sometimes the case that relevant deformations, such as those induced by rotations of the NS5 branes, lead to a flow along the manifold of fixed points in the infrared, but this is not generically the case.

Next we will consider the elliptic models with O6 planes. The $x_{6}$ direction is compact: $x_{6} \approx x_{6}+2 L$. In that case, there are two orientifold fixed planes located at the two fixed points of the action $x_{6} \rightarrow-x_{6}\left(x_{6}=0, L\right)$. The $6^{ \pm}$planes carry \pm 4 units of sixbrane charge ( \pm 2 physical units). As was observed in [6], vanishing total Ramond-Ramond sixbrane charge in Type IIA $\mathcal{N}=2$ brane configurations is necessary for finiteness of the resulting gauge theories. This is clear given the relation between NS5 brane bending and the $\beta$ function, and is equivalent to the condition for tadpole cancelation in the Type IIB picture of the same theories obtained after performing a T-duality in the $x_{6}$ direction.

For example, consider a $\mathcal{N}=2$ theory with two NS5 branes away from the orientifolds. If the two O6 planes have opposite charges, as in Fig. 26, then no D6 branes are required to cancel the sixbrane charge, and the finite theory on the D4 branes is a $\mathrm{SO}(2 N) \times \mathrm{USp}(2 N-2)$ gauge theory with two bifundamental half hypermultiplets.

We can now perform a T-duality along the $x_{6}$ direction, as in [6]. The D4 branes map to D3 branes. The two NS5 branes give a $A_{1}$ ALE space in the $\left(x_{6}, x_{7}, x_{8}, x_{9}\right)$ directions which corresponds to a $\mathbb{Z}_{2}$ singularity at $x_{6}=x_{7}=x_{8}=x_{9}=0$. The orbifold group is $\left\{1, R_{6789}\right\}$ where $R_{6789}$ is the reflection

$$
R_{6789}:\left(x_{6}, x_{7}, x_{8}, x_{9}\right) \rightarrow\left(-x_{6},-x_{7},-x_{8},-x_{9}\right)
$$


The T-dual of the pair of O6 planes is an O7 plane. The orientifold group is $\left\{1, \Omega R_{45}\right\}$, where $\Omega$ is a world-sheet parity reversal, and the action on the Chan-Paton factors is such as to produce the correct gauge group. However, for this to be consistent with the orbifold action, the correct orientifold projection should be $\left\{1, R_{6789}, \Omega R_{45}, \Omega R_{456789}\right\}$. Roughly speaking, this corresponds to an O7 plane in the $\left(x_{0}, x_{1}, x_{2}, x_{3}, x_{6}, x_{7}, x_{8}, x_{9}\right)$ directions and an O3 plane in the $\left(x_{0}, x_{1}, x_{2}, x_{3}\right)$ directions, but these orientifolds do not carry Ramond-Ramond charge. The action of the orientifold/orbifold system on the Chan-Paton factors can be determined by tadpole cancelation [6], which leads to the $\operatorname{SO}(2 N) \times \operatorname{USp}(2 N-2)$ gauge theory as before the T-duality.

We can now rotate the two NS5 branes in opposite directions. This corresponds to adding a mass term of the form $m\left(\operatorname{Tr} \Phi_{1}^{2}-\operatorname{Tr} \Phi_{2}^{2}\right)$. Integrating out the adjoint gives a marginal quartic superpotential for the rest of the matter. The Type IIB configurations corresponding to Type IIA configurations with rotated NS5 branes are obtained by a deformation of the $\mathcal{N}=2$ configuration of the type described above and in [19], with the additional complication that orientifolds are present in these theories. We do not give more explicit constructions for this case here. Supergravity descriptions for D-branes at combined orbifold and orientifold singularities were studied in [25].

It is interesting now to consider the configuration with D4 branes parallel to O4 planes (Fig. 3). It is well known that the RR charge of an O4 plane changes sign as it passes through an NS5 brane [26, 27]. The theory on the world-volume of the D4 brane is an $\mathcal{N}=2 \mathrm{SO}(2 N)$ $\times \operatorname{USp}(2 N-2)$ gauge theory, which is the same theory we obtained above with O6 planes. By a T-duality, we apparently get D3 branes with an orientifold/orbifold projection which is the same as the one obtained for the previous case. This is an interesting example of different Type IIA brane configurations which describe the same theory on the world volume of the D4 branes, and have the same T-dual in the compact direction. A puzzle arises when we consider T-dualizing the Type IIB theory along the compact direction. It is unclear which of the Type IIA configurations it should T-dualize to. These types of orientifolds are not yet understood well enough to resolve this issue, and merit further study.

There is another configuration of two NS5 branes and O6 planes on a circle, in which the NS5 branes are stuck in position to the O6 planes, giving rise to an $\mathcal{N}=2 \mathrm{SU}(2 N)$ theory with symmetric and antisymmetric tensor hypermultiplets. The T-dual of this configuration is again given by an $\mathrm{O} 7$ plane with spacetime action as above, but with a different choice of Chan-Paton matrices, as described in [6, 27, 28, 29]. Note that the configuration with the O4 plane wrapping the $x_{6}$ direction cannot give rise to this theory by changing the positions of the NS5 branes. This theory has $Q^{4}$ type marginal operators as discussed earlier, but the NS5 branes in the corresponding brane configuration (Fig. 11) are not free to rotate. In this 


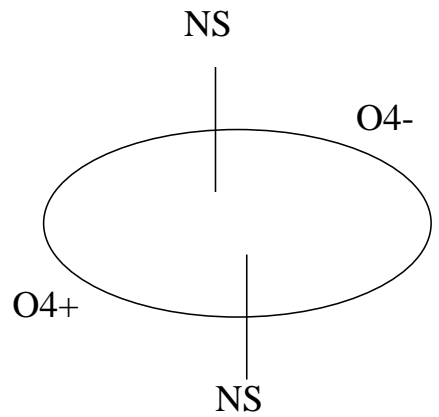

Figure 3: Brane configuration with O4 plane wrapping the compact direction.

case the only marginal deformation is from changing the circle radius.

\section{Brane configurations for theories with exactly marginal operators}

As discussed in Section 3, construction of brane configurations of intersecting NS5 branes and D4 branes with all the NS5 branes having the same linking number is a convenient way to generate theories with exactly marginal operators. In this section, we study a number of such configurations. For the configurations preserving 8 supercharges, which correspond to four dimensional $\mathcal{N}=2$ supersymmetric low energy theory on the world-volume of the D4 branes, equal linking numbers of the NS5 branes and the existence of an exactly marginal operator in the field theory is implied by vanishing one-loop $\beta$ function for the field theory (which, in this case, implies that the theory is finite). The linking number criterion for brane configurations generates almost all $\mathcal{N}=2$ finite configurations with factors of classical gauge groups. Integrating out the adjoint chiral field from the $\mathcal{N}=2$ finite theory, we find an $\mathcal{N}=1$ theory with a quartic superpotential which was shown to have an exactly marginal operator in Section 2. As we will see, some of the $\mathcal{N}=1$ field theories with quartic superpotentials that we obtain by studying brane configurations are not related to $\mathcal{N}=2$ theories by integrating out an adjoint. 


\section{1 $\mathcal{N}=2$ finite theories with classical gauge groups $\mathrm{SU}(N)$ with $2 N \square$ hypermultiplets}

The brane configuration is shown in Fig. 1. The linking number of each NS5 brane is zero, so the NS5 branes are asymptotically straight. As discussed in the previous section, there are two equivalent ways of getting the same field theory on the world volume of the D4 branes with or without D6 branes. By rotating one of the NS5 branes in the configuration with no D6 branes, we get an $\mathcal{N}=1$ configuration in which the NS5 branes still do not bend so we expect an exactly marginal operator in the $\mathcal{N}=1$ field theory on the D4 branes (which is $\mathrm{SU}(N)$ gauge theory with $2 N$ flavors). Rotating the NS5 branes corresponds to integrating out the adjoint, which results in a quartic superpotential for the field theory. The analysis in Section 2 shows that there is indeed an exactly marginal operator in the field theory.

\section{$\operatorname{USp}(2 N)$ with $(2 N+2) \square$ hypermultiplets}

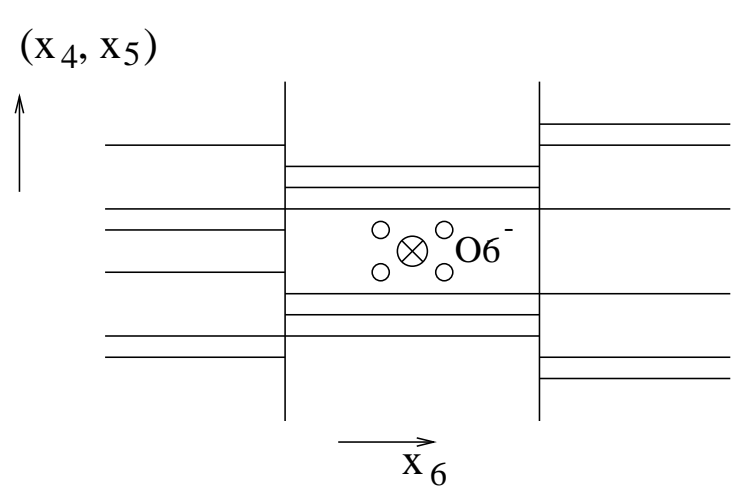

(a)

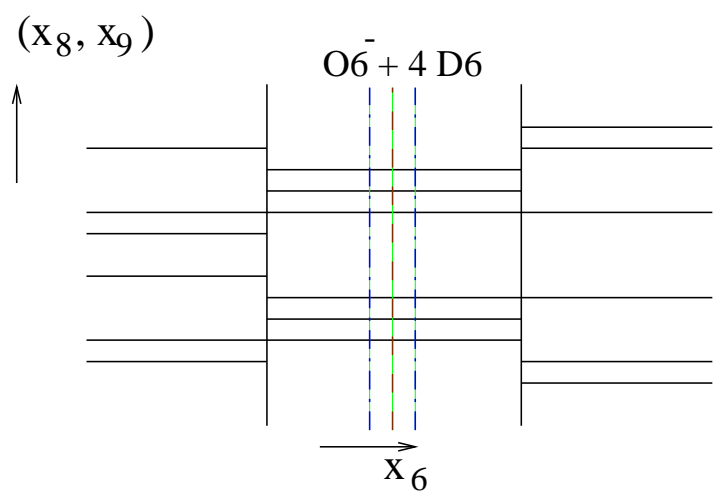

(b)

Figure 4: (a) $\mathcal{N}=2 \operatorname{USp}(2 N)$ theory with $(2 N+2) \square$ hypermultiplets. (b) $\mathcal{N}=1 \operatorname{USp}(2 N)$ gauge theory with $\mathrm{B}$ and two types of flavors, $Q$ and $f$.

The sixbrane $\mathrm{RR}$ charge of the $\mathrm{O6}^{-}$plane can be locally canceled by putting 4 D6 branes on top of it. The rest of the D6 branes can be moved in pairs, past the NS5 branes and to infinity to get a configuration with $2 N$ semi-infinite $\mathrm{D} 4$ branes on each side (Fig.(4). The two NS5 branes can then be rotated by an angle $\theta$ out of the $\left(x_{4}, x_{5}\right)$ plane into the $\left(x_{8}, x_{9}\right)$ plane. This just corresponds to integrating out the adjoint and gives a theory with an exactly marginal operator. When $\theta=\pi / 2$, an antisymmetric tensor chiral field, $A$, becomes massless and parameterizes the motion of the D4 branes along the NS5' branes (Fig.(4). This theory has two types of flavors- $2 N$ ( $4 N \square$ chiral multiplets) flavors which we call $Q$ come from 
the semi-infinite D4 branes and two flavors ( $4 N \square$ chiral multiplets), $f$ from the D6 branes. The matter content can be summarized as:

\begin{tabular}{ccc}
\multicolumn{3}{c}{$\mathrm{USp}(2 N)$} \\
\hline$A$ & $\boxminus$ & 1 \\
$Q$ & $\square$ & $4 N$ \\
$f$ & $\square$ & 4
\end{tabular}

The superpotential is

$$
W=Q A Q+Q^{4}+(Q f)^{2}+f^{4}
$$

The conditions for vanishing $\beta$ functions are:

$$
\begin{aligned}
& 0=3(2 N+2)-(2 N-2)\left(1-\gamma_{A}\right)-4 N\left(1-\gamma_{Q}\right)-4\left(1-\gamma_{f}\right) \\
& 0=\gamma_{A}+2 \gamma_{Q} \\
& 0=1+2 \gamma_{Q} \\
& 0=1+\gamma_{Q}+\gamma_{f} \\
& 0=1+2 \gamma_{f} .
\end{aligned}
$$

Only three of these equations are linearly independent implying the possibility that the theory has a two dimensional manifold of fixed points and hence has two exactly marginal operators. As before, we can identify the translations in the $x_{6}$ directions of the NS5 branes with an exactly marginal operator. However, this accounts for only one such operator. It is not easy to check if all the quartic terms in the superpotential are actually present. These terms arise from integrating out the the adjoint in the $\mathcal{N}=2$ theory. If we assume that one of these terms is zero, we will get only one exactly marginal operator from the field theory analysis which agrees with the counting from the brane picture. However, if all the quartic superpotential terms are non-zero and the field theory has two exactly marginal operators, we might be able to see the second exactly marginal operator by the motion of the 4 D6 branes in the $x_{6}$ direction. This motion is parameterized by one variable if we require that two physical D6 branes remain on top of each other when they move; from the field theory point of view, this means that the operator preserves an $\mathrm{SO}(4)$ flavor symmetry acting on the $f$ 's, which is implied by the superpotential above. Although motion of the D6 branes is irrelevant for the IR dynamics in the $\mathcal{N}=2$ case, here, we expect it to be important: for example, when we move the D6 branes past the NS5 branes, the theory loses a flavor. So motion of the D6 brane past the NS5' branes corresponds to a relevant operator for the 
field theory. We propose that the motion of the D6 branes might correspond to an exactly marginal operator when the D6 branes are between the NS5' branes.

\section{$\operatorname{SO}(N)$ with $(N-2) \square$ hypermultiplet}

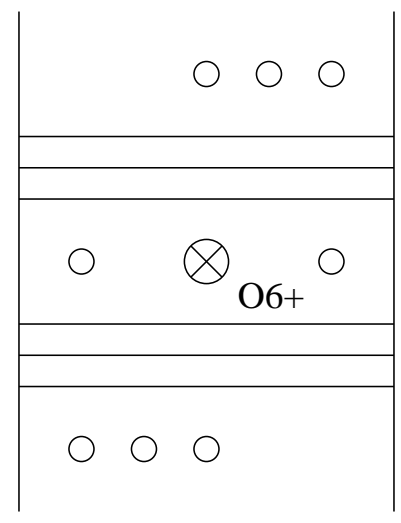

Figure 5: $\mathcal{N}=2 \mathrm{SO}(N)$ theory with $(N-2) \square$ hypermultiplets.

If $N$ is odd, there is a D4 brane which cannot move from the $\mathrm{O} 6$ plane. For the $\mathcal{N}=2$ configuration (Fig. NS5 branes from the $\left(x_{4}, x_{5}\right)$ plane into the $\left(x_{8}, x_{9}\right)$ plane by an angle $\theta$. The presence of the orientifold plane causes the image of the NS5 brane to move with an angle $-\theta$. This corresponds to integrating out the adjoint giving rise to a quartic superpotential. As discussed in Section 3, an interesting $\mathcal{N}=1$ configuration arises when $\theta=\pi / 2$. However, for the purpose of generating a field theory with an exactly marginal operator, this configuration is not very useful since the charge of the $\mathrm{O}^{+}$plane cannot be canceled.

\section{SU $(N)$ with $\square+(N-2) \square$ hypermultiplets}

The linking number of each NS5 brane is zero which implies that the NS5 branes do not bend and the $\beta$ function of the field theory on the $\mathrm{D} 4$ branes world volume is zero (Fig.6).

An interesting chiral $\mathcal{N}=1$ configuration is obtained in which the NS5 brane on top of an O6 plane is replaced by an NS5' brane in directions $\left(x_{0}, x_{1}, x_{2}, x_{3}, x_{8}, x_{9}\right)$ [30, 31]. The NS5' brane, located at $x_{7}=0$ divides the $\mathrm{O} 6$ plane into two regions $-x_{7}>0$ and $x_{7}<0$. In such a configuration, the orientifold charge jumps from -4 to +4 as we cross the NS5' brane [26]. The part of the orientifold with negative charge has 8 semi-infinite D6 branes embedded in it which are required by charge conservation [30]. Now $N$ D4 branes are stretched between an NS5 brane and its image under such an orientifold (with the NS5' brane and 8 semi-infinite 


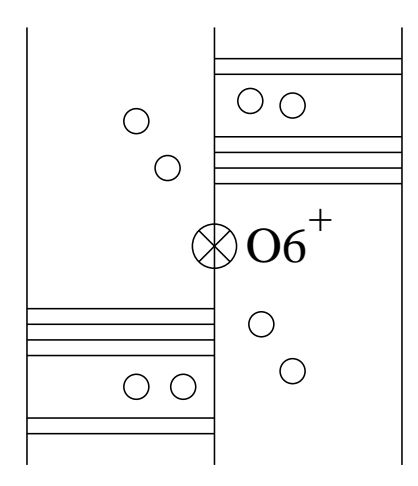

(a)

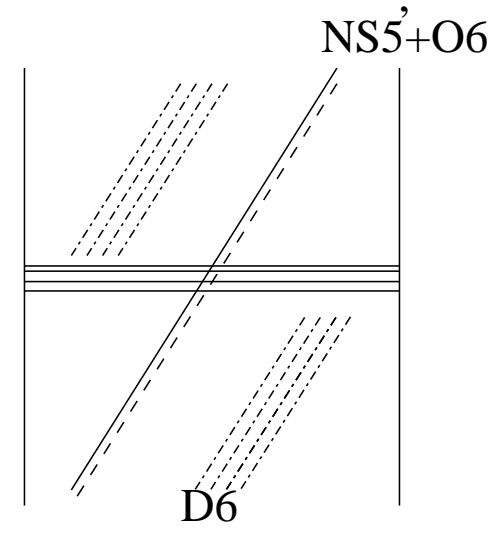

(b)

Figure 6: (a) $\mathcal{N}=2 \mathrm{SU}(N)$ with $\square$ and $(N-2) \square$ hypermultiplets. (b) Replacing the center NS5 with NS5' to give an $\mathcal{N}=1$ configuration.

D6 branes embedded in it) (Fig.6b). For calculation of the linking number, this orientifold should act exactly like an $\mathrm{O}^{+}$plane. So we need $2(N-2)$ D6 branes $(N-2$ physical D6 branes and their images) for the linking numbers of each NS5 branes to be zero. As discussed in [30], the theory on the world-volume of the $\mathrm{D} 4$ branes is a chiral $\mathcal{N}=1 \mathrm{SU}(N)$ gauge theory with chiral fields:

\begin{tabular}{c|c|c} 
& $\mathrm{SU}(N)$ & \\
\hline $\mathrm{X}$ & $\boxminus$ & 1 \\
$\tilde{S}$ & $\square$ & 1 \\
$\mathrm{Q}$ & $\square$ & $2 N+4$ \\
$\tilde{Q}$ & $\bar{\square}$ & $2 N-4$
\end{tabular}

It is easy to check that this theory is anomaly free - the total anomaly $(2 N+4)-(2 N-$ $4)+(N-4)-(N+4)$ is zero. The theory has a superpotential

$$
W=Q \tilde{S} Q+\tilde{Q} X \tilde{Q}
$$

If we rotate the NS5 branes out of the $v=x_{4}+i x_{5}$ plane and into the $w=x_{8}+i x_{9}$ plane by an angle $\theta$ and its image by $-\theta$, the theory will have an adjoint $\Phi$ which will in general be massive except when $\theta=\pi / 2$ when the adjoint becomes massless. The superpotential for 
the configuration with a generic value of $\theta$ is

$$
W=Q \tilde{S} Q+\tilde{Q} X \tilde{Q}+\Phi X \tilde{S}+\mu(\theta) \Phi^{2}
$$

For nonzero values of $\mu(\theta)$, we can integrate $\Phi$ out and obtain the superpotential

$$
W=Q \tilde{S} Q+\tilde{Q} X \tilde{Q}+\frac{1}{\mu(\theta)}(X \tilde{S})^{2}
$$

The equations for vanishing $\beta$ functions for the couplings of this theory are:

$0=3(2 N)-(N-2)\left(1-\gamma_{X}\right)-(N+2)\left(1-\gamma_{\tilde{S}}\right)-(2 N+4)\left(1-\gamma_{Q}\right)-(2 N-4)\left(1-\gamma_{\tilde{Q}}\right)$

$0=2 \gamma_{Q}+\gamma_{\tilde{S}}$

$0=2 \gamma_{\tilde{Q}}+\gamma_{X}$

$0=1+\gamma_{X}+\gamma_{\tilde{S}}$.

These equations are linearly dependent- any three imply the fourth. So we expect an exactly marginal operator in the field theory which is what we see from the brane picture.

SU $(N)$ with $\boxminus+(N+2) \square$ hypermultiplets.

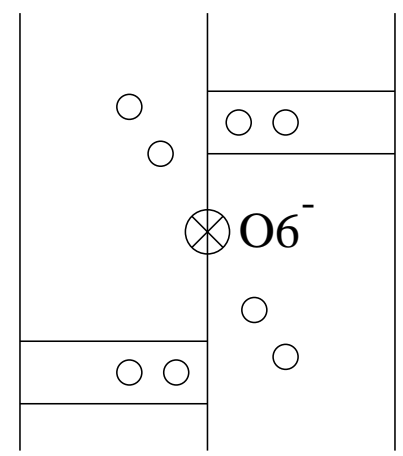

Figure 7: $\mathrm{SU}(N)$ with $\boxminus$ and $(N+2) \square$ hypermultiplets

The $\mathcal{N}=2$ configuration is the same as the previous model except that the sign of orientifold is reversed (Fig.7). 


\section{$\mathrm{SU}(N)$ with an adjoint hypermultiplet}

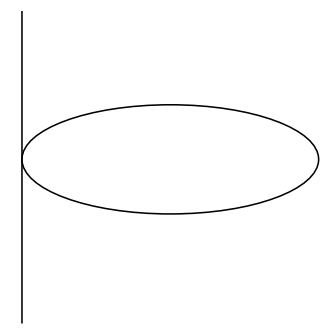

Figure 8: $\mathcal{N}=4 \mathrm{SU}(N)$ model.

This is the $\mathcal{N}=4$ theory (Fig. (8). The strings passing through the NS5 brane with end points on the $\mathrm{D} 4$ branes give rise to an adjoint hypermultiplet. There is an adjoint chiral multiplet corresponding to the motion of the D4 branes along the NS5 brane. So the matter content is indeed that on an $\mathcal{N}=4$ theory. The superpotential couplings are also exactly that of the $\mathcal{N}=4$ theory. Upon T-duality, we get $N$ D3 branes.

\section{$\mathrm{SO}(N)$ with an $\Theta$ hypermultiplet}

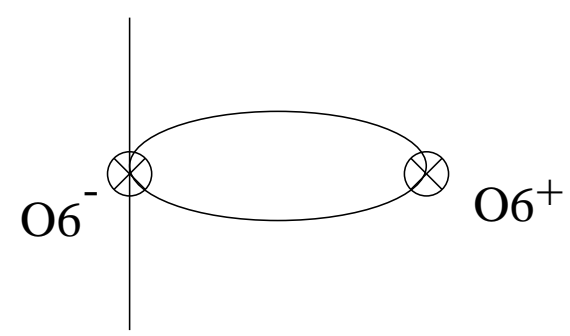

Figure 9: $\mathcal{N}=4 \mathrm{SO}(N)$ model.

This is the $\mathcal{N}=4$ theory (Fig. 9) [32]. 


\section{$\operatorname{USp}(2 N)$ with $\square$ hypermultiplet}

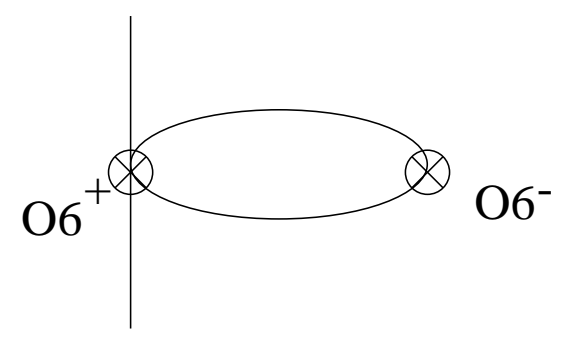

Figure 10: $\mathcal{N}=4 \operatorname{USp}(2 N)$ model.

This is an $\mathcal{N}=4$ theory (Fig.10] 32.

We cannot rotate the NS5 brane because of the orientifold symmetry. However, the configuration with an NS5' brane parallel to the ${ }^{+} 6^{+}$plane preserves 4 supercharges, and we expect to get an $\mathcal{N}=1$ theory on the D4 branes which has an exactly marginal operator. The NS5' brane splits the O6 plane into two parts and the orientifold charge jumps from -4 to +4 across the NS5' brane. As discussed above, we need 8 semi-infinite D 6 branes in the region of the orientifold with charge -4 . The field theory on the D4 branes has gauge group $\mathrm{USp}(2 N)$, and matter:

\begin{tabular}{ccc}
\multicolumn{3}{c}{$\mathrm{USp}(2 N)$} \\
\hline$Q$ & $\square$ & 8 \\
$X$ & $\boxminus$ & 1 \\
$S$ & $\square$ & 1 \\
$A$ & $\boxminus$ & 1
\end{tabular}

The theory has a superpotential

$$
W=Q S Q+A X S
$$

This theory has the matter content and couplings of the $\mathcal{N}=2$ theory and is secretly an $\mathcal{N}=2$ theory. The one loop $\beta$ function is zero and the theory is finite and has an exactly marginal operator. We can obtain this theory from a different brane configuration shown in Fig.14. 


\section{SU $(N)$ with $\theta+\square$ hypermultiplets}
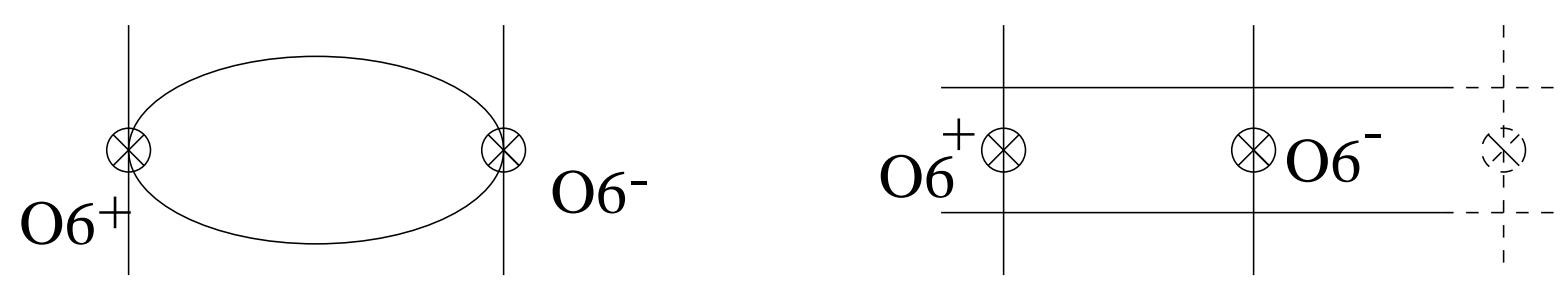

Figure 11: SU(N) with $\square$ and $\boxminus$ hypermultiplets.

The $x_{6}$ direction is compactified on a circle. It is more tricky to define linking number on a circle. Finiteness implies that the linking numbers of each NS5 branes should be the same for a fundamental domain of the circle. We cannot rotate the NS5 branes continuously because of the orientifold symmetry (Fig.11). However, there are two $\mathcal{N}=1$ configurations we can get from this theory (shown in Fig.12). In Fig.12a, the two NS5 branes are orthogonal to each other such that the NS5' brane (on top of the $\mathrm{O}^{-}$) is parallel to the O6 planes. In fact, the NS5' brane splits the orientifold into two parts (corresponding to $x_{7}>0$ and $\left.x_{7}<0\right)$ and the orientifold charge jumps from -4 to +4 at $x_{7}=0$. For charge conservation and vanishing of the six dimensional anomaly, the part of the orientifold with negative charge has eight semi-infinite D6 branes embedded in it. The theory on the D4 branes is an $\mathcal{N}=1$ theory with the following chiral fields:

\begin{tabular}{c|c} 
& $\mathrm{SU}(N)$ \\
\hline$A, \tilde{A}$ & $\boxminus, \bar{\Xi}$ \\
$\mathrm{X}$ & $\boxminus$ \\
$\tilde{S}$ & $\bar{\square}$ \\
$8 \mathrm{Q}$ & $\square$
\end{tabular}

$A$ and $\tilde{A}$ are fields associated with the strings stretching between the D4 branes on either side of the NS5 brane on top of the $\mathrm{O6}^{-}$plane. $X, \tilde{S}$ and the eight $Q$ 's come from the neighborhood of the NS5' brane on top of the O6 plane with 8 semi-infinite D6 branes stuck to it. The theory has a superpotential

$$
W=Q \tilde{S} Q+A^{4}+(X \tilde{S})^{2}
$$




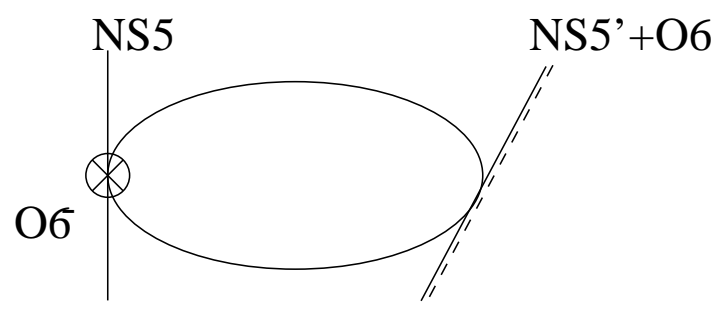

(a)

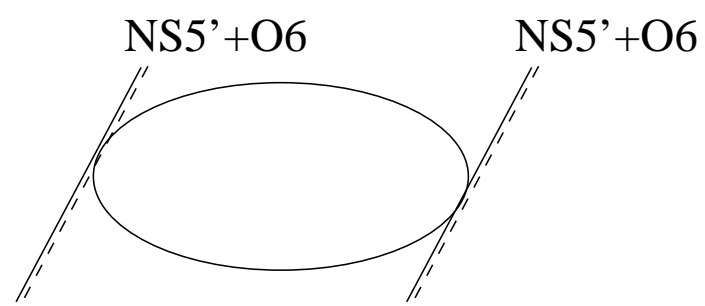

(b)

Figure 12: $\mathcal{N}=1$ configurations obtained by rotating NS5 branes in Fig.11.

The $(X S)^{2}$ term arises by integrating out the adjoint field from the $\mathcal{N}=1$ theory shown in Fig.12]b since that theory has a coupling of the form $X \Phi S$. Going from configuration in Fig. 12b to that in Fig 12a involves giving a mass to $\Phi$. The $A^{4}$ term arises by integrating out the adjoint from the $\mathcal{N}=2$ theory. The conditions for all $\beta$ functions to vanish are:

$$
\begin{aligned}
& 0=3(2 N)-8-3(N-2)-(N+2)+8 \gamma_{Q}+2(N-2) \gamma_{A}+(N-2) \gamma_{X}+(N+2) \gamma_{\tilde{S}} \\
& 0=2 \gamma_{Q}+\gamma_{\tilde{S}} \\
& 0=1+2 \gamma_{A} \\
& 0=1+\gamma_{X}+\gamma_{\tilde{S}} .
\end{aligned}
$$

It is easy to see that these equations are linearly dependent. There should be one exactly marginal operator (according to the analysis in section 2).

\section{SU $(N)$ with 2 日 and $4 \square$ hypermultiplets}

The orientifolds are $\mathrm{O}^{-}$planes which have -4 units of sixbrane $\mathrm{RR}$ charge. Finiteness of the field theory implies that the RR charge in the $x_{6}$ direction vanishes and that is achieved by the addition of 8 D6 branes (4 physical branes and 4 images). These D6 branes give 4 $\square$ hypermultiplets. The NS5 branes on each $\mathrm{O}^{-}$planes gives rise to 2 日 hypermultiplets.

The $\mathcal{N}=1$ configurations which correspond to NS5 branes being replaced by NS5' are not interesting for our purposes because they will necessarily have non-vanishing sixbrane RR charge. The orientifold charge jumps from -4 to +4 where it intersects the NS5' brane. As explained above, we need 8 semi-infinite D6 branes embedded in the side of the orientifold 

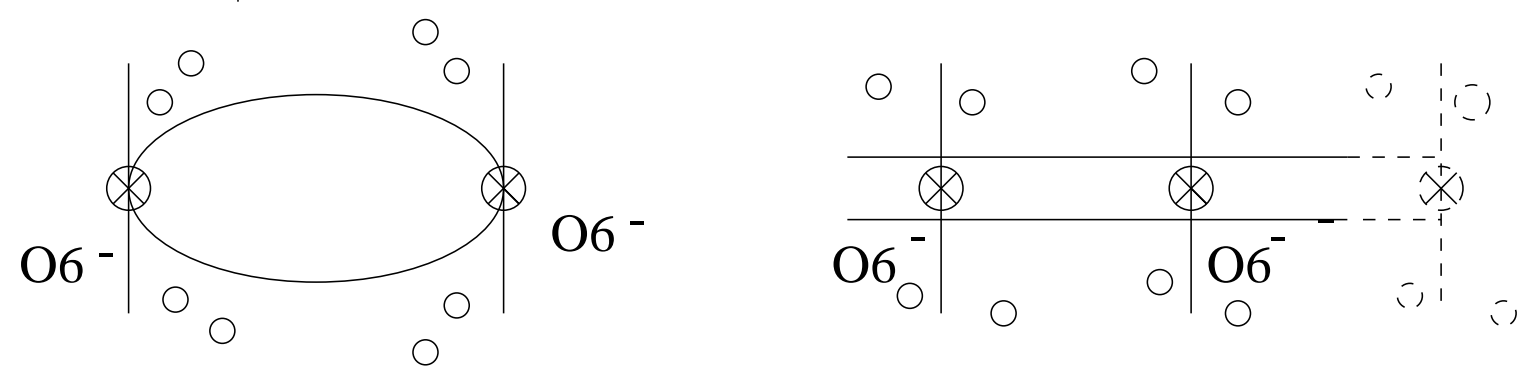

Figure 13: $\mathrm{SU}(N)$ with 2 日 and $4 \square$ hypermultiplets.

with negative charge. So the orientifold plane with 8 semi-infinite D6 branes embedded has RR sixbrane charge +4 .

USp( $2 N)$ with $日$ and $4 \square$ hypermultiplets

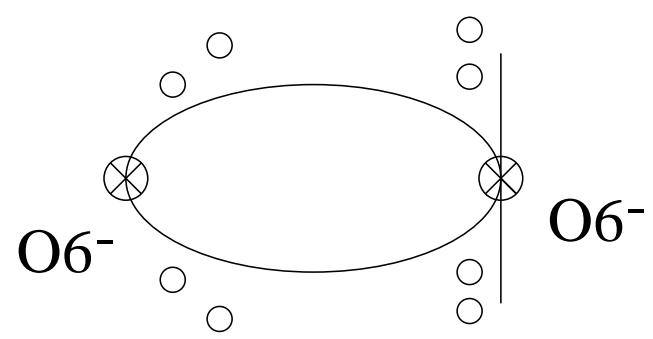

Figure 14: USp(2N) with $\Theta$ and $4 \square$ hypermultiplets.

We need four physical D6 branes to cancel the sixbrane RR charge of the orientifolds. The T-dual is D3 branes with 4 D7 branes and an $\mathrm{O}^{-}$plane. This has been discussed in 33.

\subsection{Product group theories with two factors of simple groups}

In this section, we construct brane configurations for product group theories which have manifolds of fixed points. For simplicity, we only draw the $\mathcal{N}=2$ configurations. Different 
ways of rotating branes in the $\mathcal{N}=2$ configurations in general lead to different $\mathcal{N}=1$ theories which can be analyzed by the tool developed in the previous section.

$\mathbf{S U}(N) \times \mathbf{S U}(M)$ with matter content:

\begin{tabular}{ccc}
$\mathrm{SU}(N)$ & $\mathrm{SU}(M)$ & \\
\hline$\square$ & $\square$ & 1 \\
$\square$ & 1 & $2 N-M$ \\
1 & $\square$ & $2 M-N$ \\
\hline
\end{tabular}

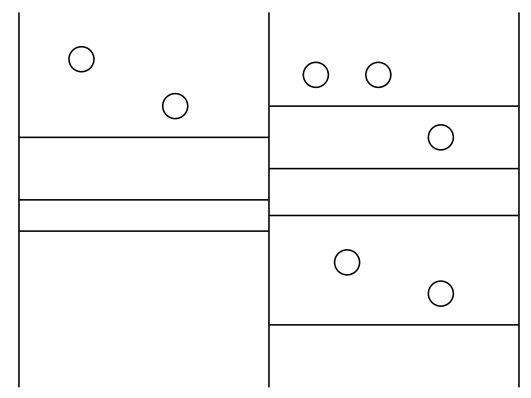

Figure 15: $\mathrm{SU}(N) \times \mathrm{SU}(M)$ with $(\square, \square)$ and flavors under each group.

The linking number of each NS5 branes is $(M-N) / 2$ so the field theory is finite (Fig. 1.5). Rotating one of the NS5 branes gives a $\mathcal{N}=1$ theory with a quartic superpotential. Rotating the middle NS5 brane corresponds to the theory with both adjoints integrated out, while rotating one of the outer NS5 branes corresponds to integrating out only one of the adjoints.

$\mathbf{S U}(N) \times \mathbf{S U}(M)$ with matter hypermultiplets:

\begin{tabular}{ccc}
$\mathrm{SU}(N)$ & $\mathrm{SU}(M)$ & \\
\hline$\square$ & $\square$ & 1 \\
$日, \square$ & 1 & 1 \\
$\square$ & 1 & $N-M \pm 2$ \\
1 & $\square$ & $2 M-N$ \\
\hline
\end{tabular}

This model involves putting an NS5 brane on top of the $6^{ \pm}$plane (Fig.16). The linking number of each NS5 brane is 0 precisely when the number of D6 branes and D4 branes are equal to the numbers predicted from the vanishing $\beta$ functions for the field theory. For O6 $^{-}$, we get an $甘$ under $\mathrm{SU}(N)$; for $\mathrm{O6}^{-}$, we get a $\square$ under $\mathrm{SU}(N)$. 


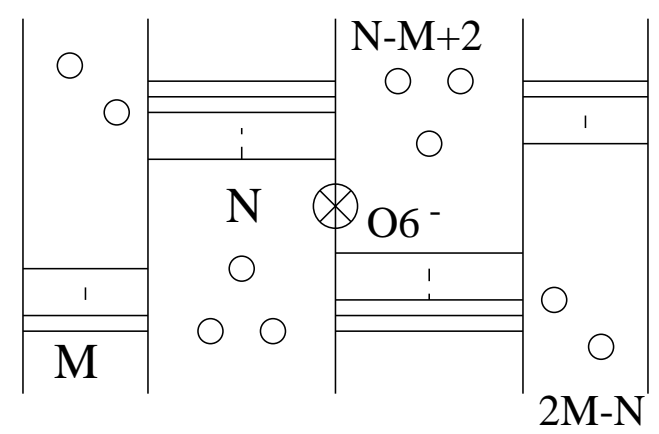

Figure 16: $\mathrm{SU}(N) \times \mathrm{SU}(M)$ with $(\boxminus, 1)$ and $\square$ hypermultiplets under each group. $\mathbf{S U}(N) \times \mathbf{S U}(N+2)$ with hypermultiplets:

\begin{tabular}{cc}
$\mathrm{SU}(N)$ & $\mathrm{SU}(N+2)$ \\
\hline$\square$ & $\square$ \\
$日$ & 1 \\
1 & $\square$
\end{tabular}

This is shown in Fig.[17

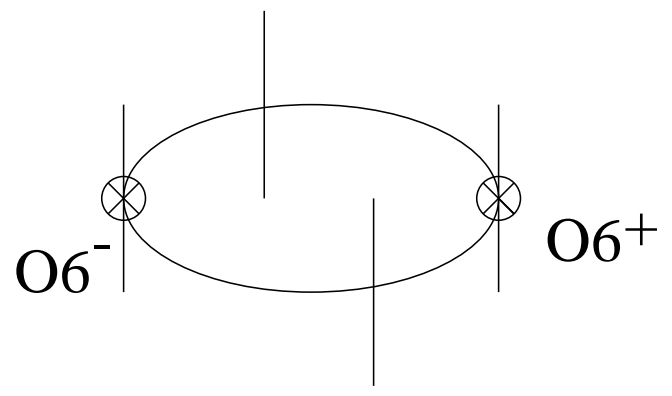

Figure 17: $\mathrm{SU}(N) \times \mathrm{SU}(N+2)$ with $(\square, \square)$ and $(\boxminus, 1)$ and $(1, \square)$ 
$\mathbf{S U}(N) \times \mathbf{S U}(M)$ with hypermultiplets:

\begin{tabular}{ccc}
$\mathrm{SU}(N)$ & $\mathrm{SU}(M)$ & \\
\hline$\square$ & $\square$ & 1 \\
$日$ & 1 & 1 \\
$\square$ & 1 & $N-M+2$ \\
1 & $\mathrm{G}$ & 1 \\
1 & $\square$ & $M-N+2$ \\
\hline
\end{tabular}

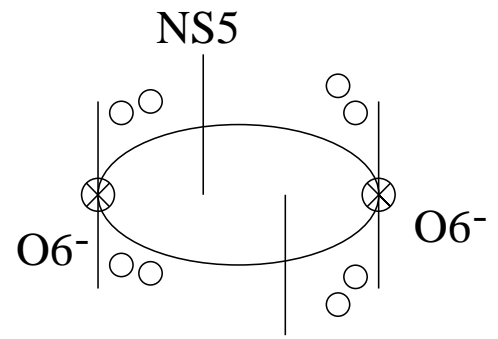

Identified

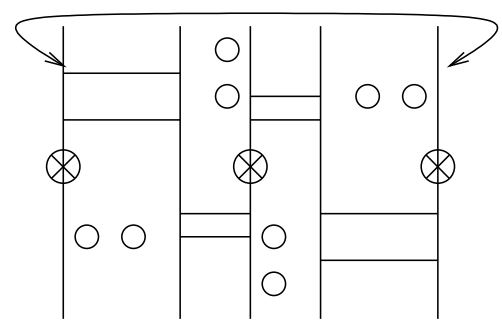

(a)

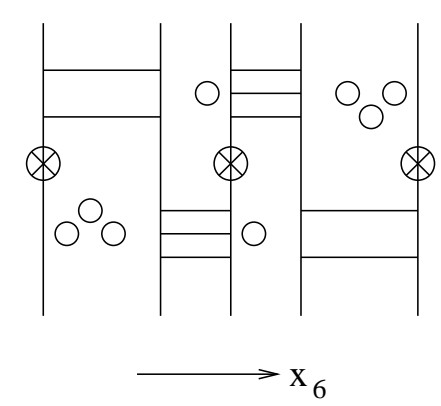

(b)

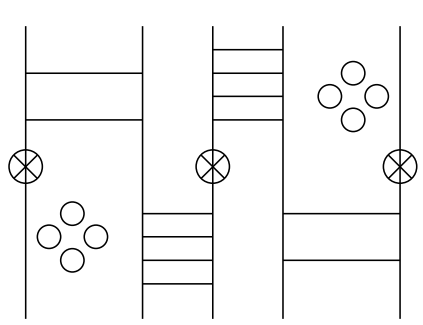

(c)

Figure 18: $\mathrm{SU}(N) \times \mathrm{SU}(M)$ with $(\square, \square)$ and $\boxminus$ and $\square$ under each group: (a), (b) and (c) are examples of cases when $|N-M|=0,1,2$.

Here, $|N-M| \leq 2$. For each value of $|N-M|$, it can be easily shown that the linking numbers of the NS5 branes are the same (Fig.18). 
$\mathbf{S U}(N) \times \mathbf{S U}(N)$ with hypermultiplet:

\begin{tabular}{ccc}
$\mathrm{SU}(N)$ & $\mathrm{SU}(N)$ & \\
\hline$\square$ & $\square$ & 1 \\
$\square$ & $\square$ & 1 \\
\hline
\end{tabular}

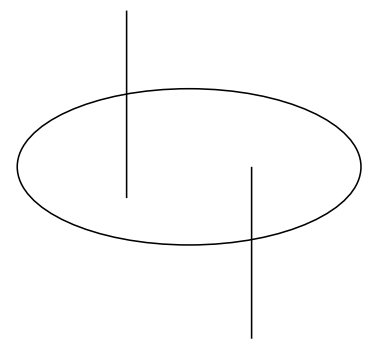

Figure 19: $\mathrm{SU}(N) \times \mathrm{SU}(N)$ with two $(\square, \square)$ hypermultiplets.

This is the elliptic model with no orientifolds and D6 branes. The linking number of each NS5 brane is 0 because there is an equal number of D4 branes to the left and right of each NS5 brane (Fig.19).

$\mathbf{S O}(N) \times \mathbf{S U}(M)$ with hypermultiplets:

\begin{tabular}{ccc}
$\mathrm{SO}(N)$ & $\mathrm{SU}(M)$ & \\
\hline$\square$ & $\square$ & 1 \\
$\square$ & 1 & $N-M-2$ \\
1 & $\square$ & $2 M-N$ \\
\hline
\end{tabular}

This is shown in Fig.20.

$\mathbf{U S p}(2 N) \times \mathbf{S U}(M)$

\begin{tabular}{ccc}
$\mathrm{USp}(2 N)$ & $\mathrm{SU}(M)$ & \\
\hline$\square$ & $\square$ & 1 \\
$\square$ & 1 & $2 N-M+2$ \\
1 & $\square$ & $2 M-2 N$ \\
\hline
\end{tabular}

This is the same as the previous case except the sign of the orientifold is reversed Fig.21. 


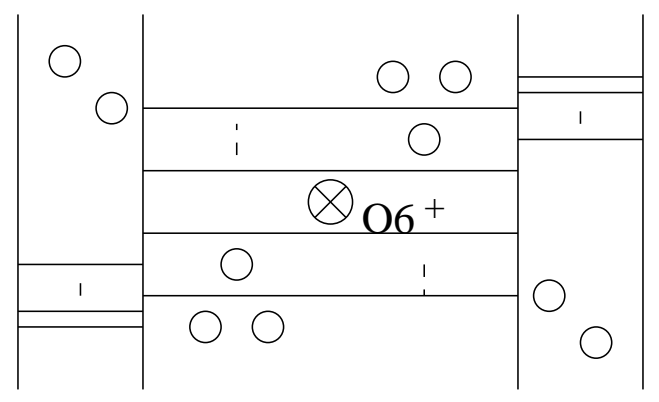

Figure 20: $\mathrm{SO}(N) \times \mathrm{SU}(M)$ with $(\square, \square)$ and flavors.

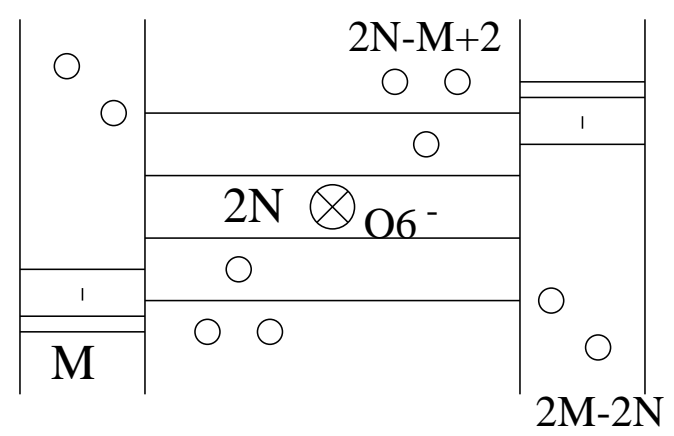

Figure 21: $\operatorname{USp}(2 N) \times \operatorname{SU}(M)$ with $(\square, \square)$ and flavors

$\mathbf{S O}(N) \times \mathbf{S U}(N-2)$

\begin{tabular}{ccc}
$\mathrm{SO}(N)$ & $\mathrm{SU}(N-2)$ & \\
\hline$\square$ & $\square$ & 1 \\
1 & $\boxminus$ & 1 \\
\hline
\end{tabular}

The linking numbers of the NS5 branes are the same (Fig.22).

$\mathbf{U S p}(2 N) \times \mathbf{S U}(2 N+2)$

\begin{tabular}{ccc}
$\mathrm{USp}(2 N)$ & $\mathrm{SU}(2 N+2)$ & \\
\hline$\square$ & $\square$ & 1 \\
1 & $\square$ & 1 \\
\hline
\end{tabular}




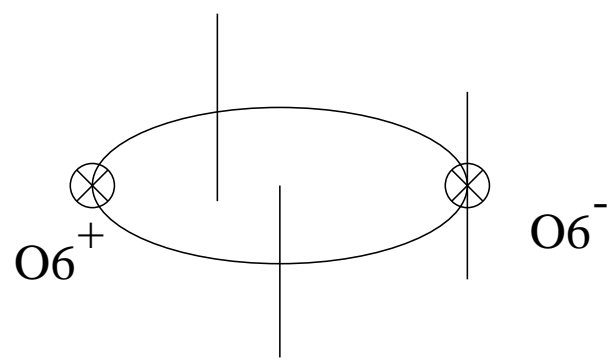

Figure 22: $\mathrm{SO}(N) \times \mathrm{SU}(N-2)$ with $(\square, \square)$ and $(1, \boxminus)$.

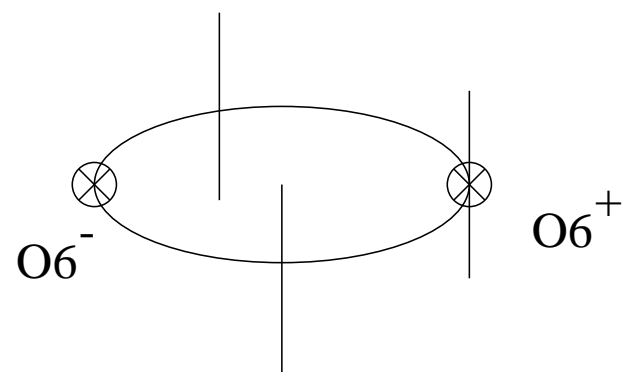

Figure 23: $\operatorname{USp}(2 N) \times \operatorname{SU}(2 N+2)$ with $(\square, \square)$ and $(1, \square)$.

This is again an elliptic model — the signs of the orientifolds are reversed compared to the previous theory (Fig.23).

$\mathbf{U S p}(2 N) \times \mathbf{U S p}(2 M)|2 M-2 N| \leq 2$

\begin{tabular}{ccc}
$\mathrm{USp}(2 N)$ & $\mathrm{USp}(2 M)$ & $|2 M-2 N| \leq 2$ \\
\hline$\square$ & $\square$ & 1 \\
$\square$ & 1 & $2 N-2 M+2$ \\
1 & $\square$ & $2 M-2 N+2$
\end{tabular}

This theory is shown in Fig.24. 


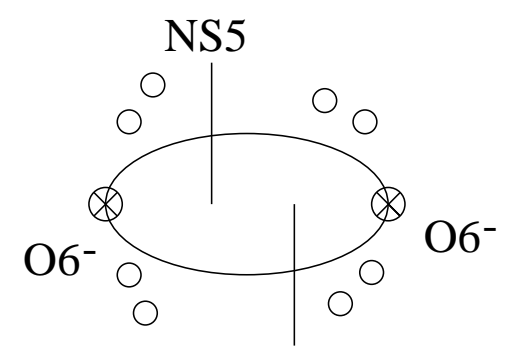

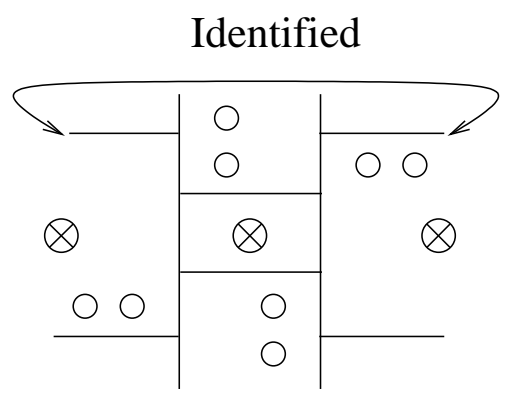

(a)

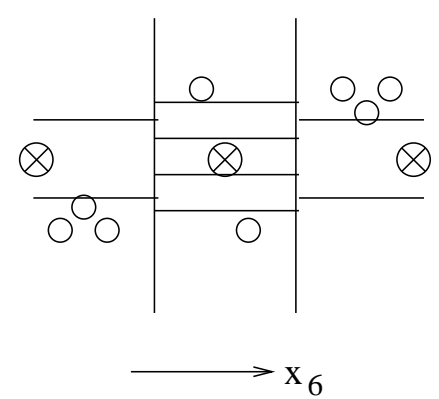

(b)

Figure 24: $\operatorname{USp}(2 N) \times \operatorname{USp}(2 M)$ with $(\square, \square), \square$ under each group:(a), (b) are the cases when $|2 N-2 M|=0,2$.

$\mathbf{U S p}(2 N) \times \mathbf{S U}(M)|M-2 N| \leq 2$

\begin{tabular}{ccc}
$\mathrm{USp}(2 N)$ & $\mathrm{SU}(\mathrm{M})$ & $|M-2 N| \leq 2$ \\
\hline$\square$ & $\square$ & 1 \\
$\square$ & 1 & $2 N-M+2$ \\
1 & $\square$ & $M-2 N+2$ \\
1 & $\boxminus$ & 1 \\
\hline
\end{tabular}

This model has two $\mathrm{O}^{-}$plane and has $8 \mathrm{D} 6$ branes so the net sixbrane $\mathrm{RR}$ charge vanishes. $|M-2 N| \leq 2$ and the different values of $|M-2 N|$ just correspond to placing the 8 D6 branes in various ways in between the NS5 branes such that the linking numbers of each NS5 brane still comes out the same (Fig.25). 


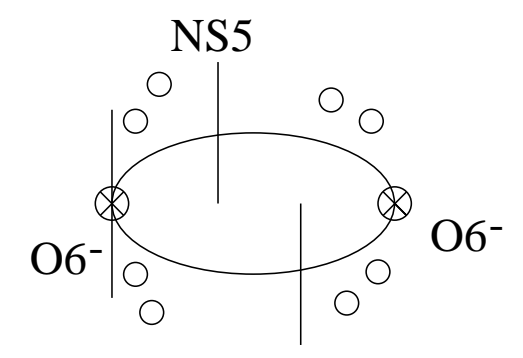

Identified

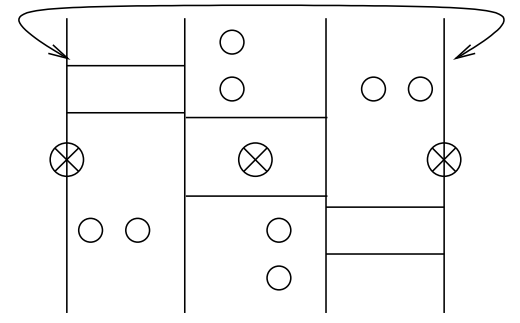

(a)

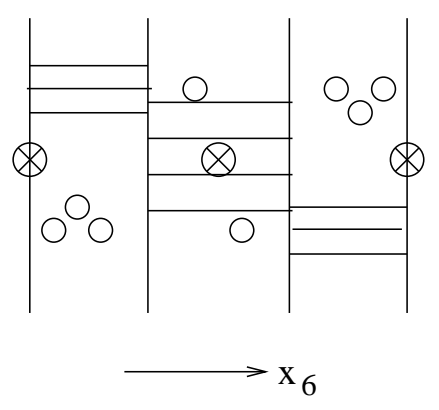

(b)

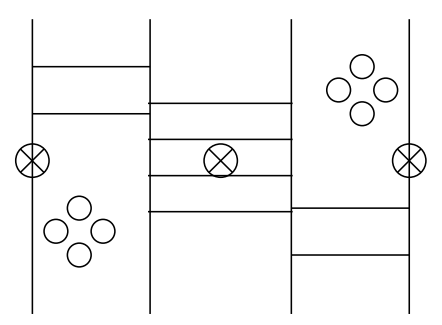

(c)

Figure 25: $\operatorname{USp}(2 N) \times \mathrm{SU}(M)$ with $(\square, \square), \square$ under each group and 日 under $\mathrm{SU}(M)$ : (a), (b) and (c) are the cases when $|2 N-M|=0,1,2$.

$\operatorname{SO}(N) \times \mathbf{U S p}(N-2)$ with half-hypermultiplets

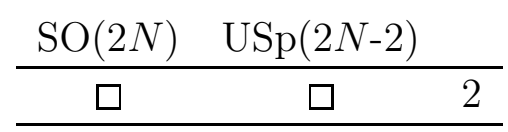

The field theory has two half-hypermultiplets. The brane configuration is in Fig. 26.

\section{$5 \quad$ Supergravity Descriptions}

As discussed in section 3, we can perform a T-duality along the compact direction for the elliptic models in Type IIA to get to a configuration with D3 branes in some singular geometry. Given the Type IIB constructions, one can in principle determine a supergravity description for those theories. This is complicated in practice because of the orbifold,conifold and orientifold geometries, but the near horizon geometry of the D3 branes in these backgrounds is expected to be related to the corresponding gauge theories via the AdS/CFT 


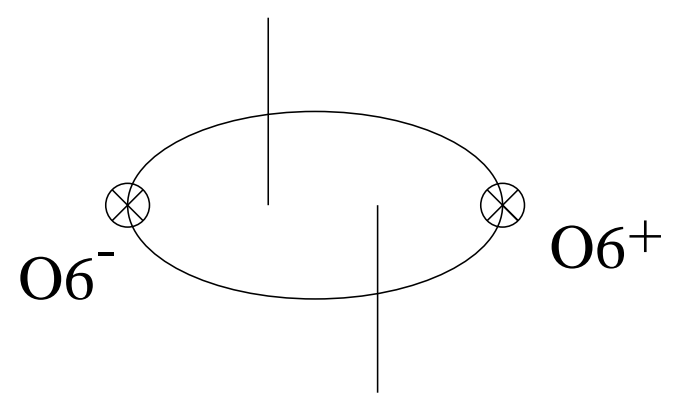

Figure 26: $\operatorname{SO}(2 N) \times \operatorname{USp}(2 N-2)$ with two $(\square, \square)$ half-hypermultiplets.

correspondence [10, 34, 35]. It has been argued [7, 9] that the difference between the Weyl and Euler anomalies must vanish to leading order in $N$ in gauge theories which have useful supergravity descriptions. In this section we argue that the elliptic models admit useful supergravity descriptions, while non-elliptic $\mathcal{N}=2$ finite and their descendent $\mathcal{N}=1$ marginal theories do not. Consider the $\mathcal{N}=2$ finite elliptic model given by the brane construction in Fig. 27. The gauge theory on the four-branes has gauge group $\mathrm{SU}(N)^{M}$ with bifundamental hypermultiplets as below:

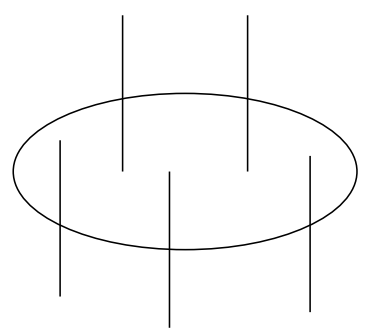

Figure 27: Brane configuration for the elliptic $\mathrm{SU}(N)^{M}$ theory.

\begin{tabular}{ccccc|c}
$\mathrm{SU}(N)$ & $\mathrm{SU}(N)$ & $\mathrm{SU}(N)$ & $\cdots$ & $\mathrm{SU}(N)$ & $\mathrm{U}(1)_{R}$ \\
\hline$\square$ & $\square$ & & & & 0 \\
& $\square$ & $\square$ & & & 0 \\
& & $\cdots$ & & & 0 \\
$\square$ & & & & $\square$ & 0 \\
\hline
\end{tabular}


Since the fermion in the adjoint chiral multiplets should transform with the same $R$-charge as the gauginos (by $\mathcal{N}=2$ supersymmetry), the adjoint chiral multiplets have $R$-charge 2 .

At a conformal fixed point, the difference between the Weyl and Euler anomalies, $c-a$, is proportional to the $\mathrm{U}(1)_{R}$ anomaly [36, 37]. The $\mathrm{U}(1)_{R}$ anomaly is easily computed:

$$
\partial\left\langle J_{R} T T\right\rangle \sim \sum_{i} \operatorname{dim} R_{i}\left(r_{i}-1\right)
$$

where $\operatorname{dim} R_{i}$ is the dimension of the representation of the chiral multiplet with $R$-charge $r_{i}$, and $T$ is shorthand for the stress tensor. Since the adjoint fermions in the $\mathcal{N}=2 \mathrm{SU}(N)^{M}$ elliptic model have charge +1 and the matter fermions have charge -1 , it is easy to see that the condition $c-a=0$ is satisfied. One way to see this is that the number of adjoint fermion degrees of freedom is $2 M N^{2}$, which is the same as the number of matter fermion degrees of freedom.

Now consider rotating one of the NS5 branes. This breaks $\mathcal{N}=2$ to $\mathcal{N}=1$ with the result of assigning $R$-charge zero to the adjoint fermions (except the gauginos, which have charge 1 by convention) and $R$-charge $-1 / 2$ to the matter multiplet fermions. The anomaly $c-a$ is proportional in this case to the number of gauginos minus half the number of matter fermion degrees of freedom. Since now the adjoint chiral fermions (half of the $\mathcal{N}=2$ vector multiplet) do not contribute, the anomaly is proportional to the $\mathcal{N}=2$ result, so $c-a=0$ in this case as well. This way of thinking about the quantity $c-a$, as counting fermion degrees of freedom, is useful because it can be easily generalized to the more complicated cases with orientifold planes.

If we add a pair of $\mathrm{O} 6$ planes in the $\mathcal{N}=2$ elliptic model with appropriate symmetry to accommodate the $\mathrm{O} 6$ reflections as described in the previous sections, the effect is as follows:

a) If the number of NS5 branes (including the images under the O6 reflection) is even, $N_{5}=2 M$, and the O6 planes do not intersect any of the NS5 branes (Fig. 28a), then the gauge theory has $M+1$ factors. Two of the factors are SO or USp, while the rest are SU. To order $N^{2}$ the adjoint of $\operatorname{SO}(N)$ or $\operatorname{USp}(N)$ has $N^{2} / 2$ degrees of freedom. Summing the contribution from the two SO or USp factors gives $N^{2}$, the same as the contribution from adjoints of the $\mathrm{SU}(N)^{M}$ elliptic model. Including the $M$ bifundamentals, the $\mathrm{U}(1)_{R}$ anomaly again vanishes.

b) If the number of NS5 branes is even, $N_{5}=2 M$, and the O6 planes intersect two of the NS5 branes (Fig. 28b), then the gauge theory has $M$ SU factors, $M-1$ bifundamentals and two symmetric or antisymmetric tensors. The symmetric and antisymmetric tensors make up the difference in degrees of freedom corresponding to the extra bifundamental in the elliptic $\mathrm{SU}(N)^{M}$ theory. 


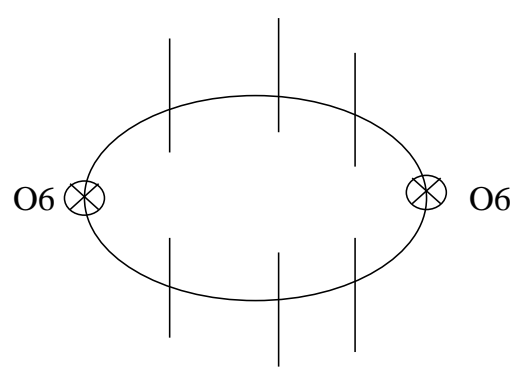

(a)

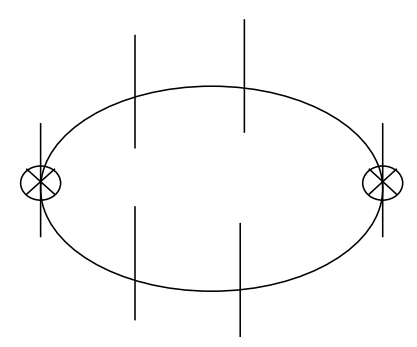

(b)

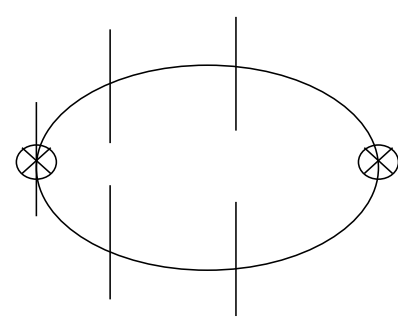

(c)

Figure 28: Elliptic models with O6 planes. D6 branes and the signs of the RR charge carried by the $\mathrm{O} 6$ planes is not given in the figures. The net D6 brane charge always cancels in these theories. Furthermore, the NS5 branes that are not stuck to the O6 planes can be rotated symmetrically.

c) IF the number of NS5 branes is odd, $N_{5}=2 M+1$, then one O6 plane intersects an NS5 brane and the other does not (Fig. 28c). The gauge theory has $M+1$ factors, one of which is SO or USp. There are $M$ bifundamentals and one symmetric or antisymmetric tensor. Since the gauginos of SO contribute half as many degrees of freedom as those of $\mathrm{SU}$, and similarly for the symmetric or antisymmetric tensor, the contribution of the SO or USp gauginos to $c-a$ cancels with the tensor, and the counting of the remaining degrees of freedom is again like the $\mathrm{SU}(N)^{M}$ theory.

Depending on the sign of the RR charges of the orientifolds, in some theories extra D6 branes will be required to cancel the flux of sixbrane charge in the $x_{6}$ direction, or equivalently for finiteness of the $\mathcal{N}=2$ theory. The D6 branes give rise to additional flavors, which are also required in those cases for conformality. The additional flavors do not contribute to $c-a$ to leading order in $N$, so they were ignored in the counting above.

Alternatively, an O4 plane can wrap the $x_{6}$ direction, parallel to the D4 branes, in which case the theory is an alternating $\mathrm{SO} \times \mathrm{USp} \times \cdots$ theory with bifundamental half hypermultiplets. It is easy to see that $c-a=0$ in this case, as well.

As discussed earlier, NS5 branes can be rotated in the orientifold theories in such a way as to preserve the orientifold symmetry, breaking $\mathcal{N}=2$ to $\mathcal{N}=1$. The argument regarding rotating branes in the $\mathrm{SU}(N)^{M}$ elliptic models is valid in these cases as well, and we find $c-a=0$ for the $\mathcal{N}=1$ elliptic orientifold models.

For certain specific orientations of the NS5 branes with respect to the O6 planes, additional massless degrees of freedom appear. For example, as described in the last section, if 
an NS5 brane and its image are parallel to the $\mathrm{O}^{ \pm}$plane, and there are no other NS5 branes between them, then an additional antisymmetric (symmetric) tensor appears for the corresponding USp (SO) gauge group, associated with movement of the D4 branes in the $x_{7}, x_{8}$ direction [23]. The additional tensor does not contribute to the $\mathrm{U}(1)_{R}$ anomaly because by gauge anomaly freedom the fermion in the tensor chiral multiplet has vanishing $R$-charge.

The equivalence of the Weyl and Euler anomalies in the elliptic theories is not surprising, since Type IIB configurations have been constructed which describe them, from which one can determine their supergravity description. However, this result can be used to prove that non-elliptic conformal or marginal theories which have a Type IIA brane description with vanishing net sixbrane charge and linking numbers do not satisfy the condition $c-a=0$. The argument is as follows: Two copies of the Type IIA brane configuration for the non-elliptic theory can be connected to form an elliptic model considered above if the net sixbrane charge and NS5 linking numbers vanish for each copy separately (Fig. 29). The resulting elliptic model satisfies the condition $c-a=0$ as discussed above. The difference in the counting of

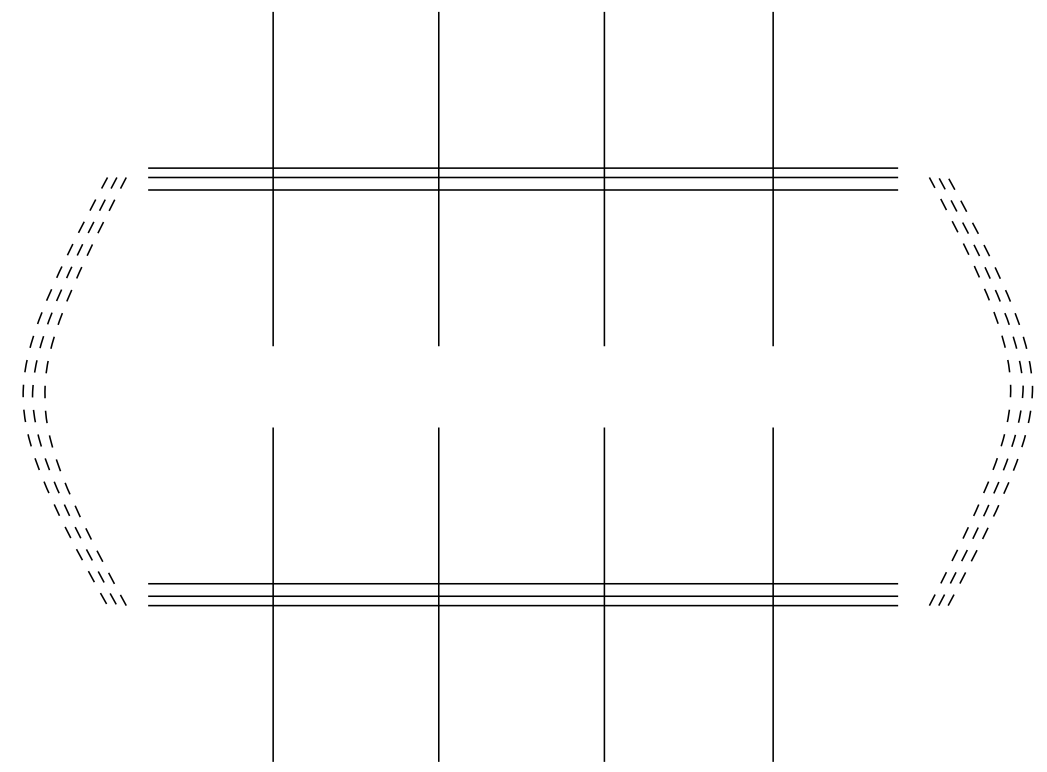

Figure 29: Glueing together copies of non-elliptic theories with vanishing net sixbrane charge on a circle.

degrees of freedom in this case versus the case of the two separate copies is from additional gauginos for each of the two new fourbrane links. Since the degrees of freedom of the new gauginos do not cancel the four additional bifundamentals, the non-elliptic theory from which we started could not have satisfied $c-a=0$. Theories which are not included in this argument include those with non-vanishing linking numbers, i.e. bending branes, theories 
with non-vanishing net sixbrane charge, and theories without semi-infinite D4 branes at both the left and right side of the configuration (Fig. 30).

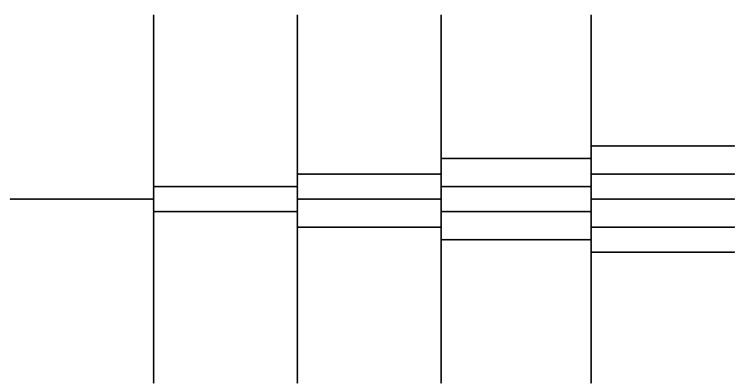

(a)

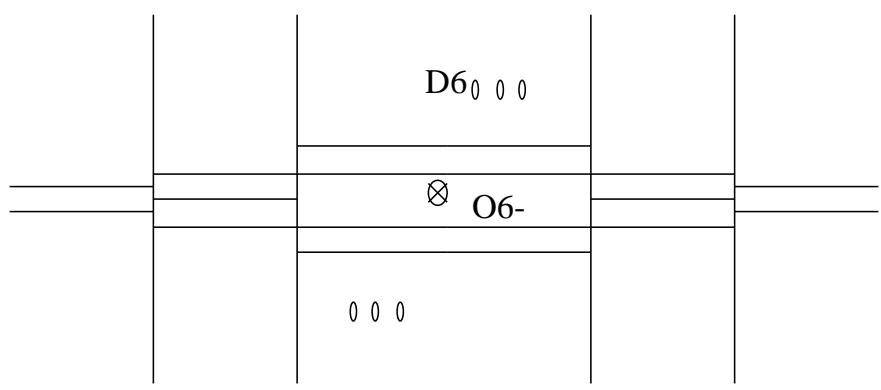

(b)

Figure 30: Some non-elliptic brane configurations not included in the discussion of Weyl and Euler anomalies.

\section{A comment on brane boxes}

A similar analysis to that of the last section can be done for brane box models. A large class of brane box models describes finite $\mathcal{N}=4,2,1$ theories [38]. In these theories there are bifundamentals and/or adjoints with cubic superpotentials. The $\mathcal{N}=2$ theories are the same as the $\mathrm{SU}(N)^{M}$ theories described above, and the $\mathcal{N}=4$ theory is the usual $\mathrm{SU}(N)$ gauge theory. The $\mathcal{N}=1$ models differ from ours in both matter content and superpotential. We briefly review their construction and describe the restrictions on which of these theories may have a supergravity description in light of the results of [7], in analogy with the discussion in the previous section.

The basic brane box for four dimensional gauge theories is a Type IIB brane configuration consisting of a two dimensional lattice of NS5 branes filled with D5 branes of finite extent in two directions. We will consider elliptic brane box configurations, in which the configuration is defined on a torus. There are two classes of such configurations:

a) $\mathcal{N}=1$ configurations can be obtained by twisting the torus of an $\mathcal{N}=2$ configuration, as in Fig. 31. There are bifundamental chiral multiplets between pairs of neighboring and diagonally neighboring $\mathrm{SU}(N)$ group factors labeled $1,2, \ldots, M$. The grid represents the NS5 branes, and there are $N$ D5 branes in each box. The matter content can be summarized as three sets of bifundamental chiral multiplets cyclically permuted among the $\mathrm{SU}(N)$ fac- 


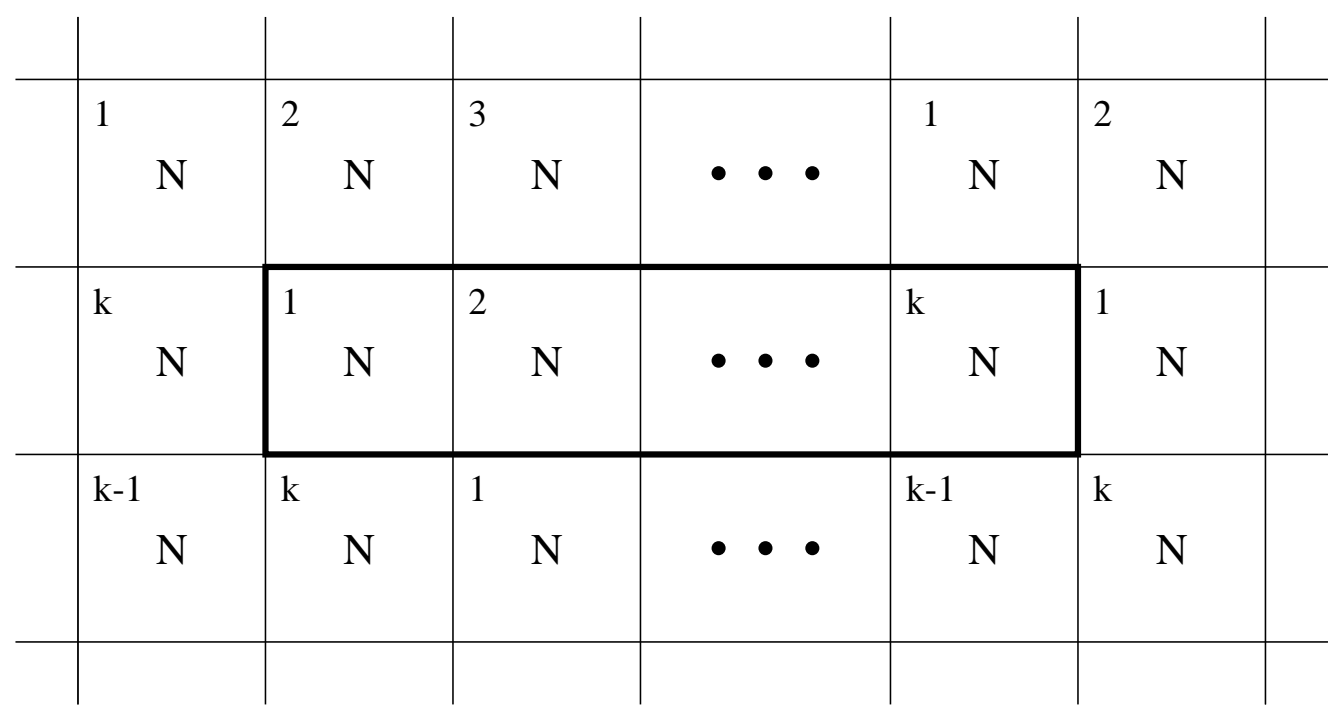

Figure 31: Twisted $1 \times k$ elliptic brane box models. There are $N$ D5 branes in each box of NS5 branes.

tors. There is a cubic superpotential consisting of gauge invariant triple products of the bifundamentals.

b)Alternatively, generic tori of $k \times k^{\prime}$ boxes with $k, k^{\prime}>1$ describe $\mathcal{N}=1$ theories, as in Fig. 32.

In either case, the number of bifundamentals is $3 M$, where $M$ is the number of boxes. Each bifundamental fermion has $R$-charge $-1 / 3$ in this case because of the cubic superpotential, so the contribution to $c-a$ of the $M N^{2}$ gaugino degrees of freedom cancels that of the $3 M N^{2}$ matter fermions, so $c-a=0$ in these elliptic brane box models. It is not surprising that the elliptic brane box models should satisfy the supergravity condition since they have T-dual descriptions in terms of D3 branes at orbifold singularities [38, 39, 40, 41].

If some of the boxes are made infinitely large in one direction, we obtain the cylindrical brane box models described in [38]. Arguments similar to those in the previous section show that these theories do not satisfy the $c-a=0$ condition.

\section{Conclusions}

We have studied four dimensional $\mathcal{N}=1$ theories with quartic superpotentials and their brane description in Type IIA and Type IIB string theories. These theories can be obtained 


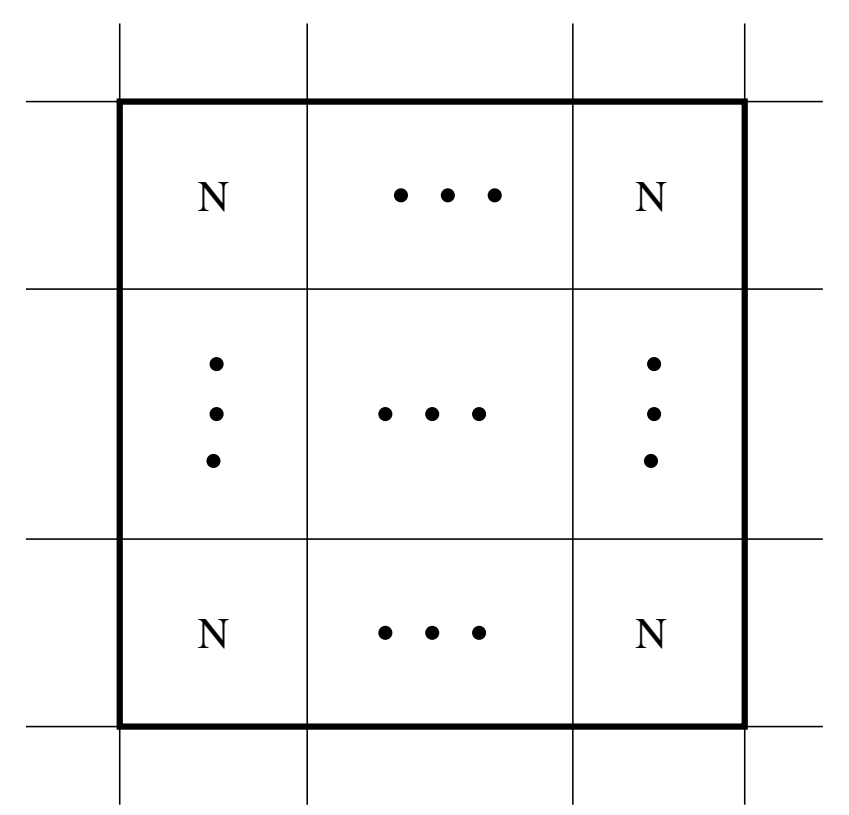

Figure 32: Generic $k \times k^{\prime}$ brane box configuration.

from $\mathcal{N}=2$ theories by integrating out the adjoint chiral multiplet. If the $\mathcal{N}=2$ theory is finite, then the resulting $\mathcal{N}=1$ theory has marginal deformations along a line of fixed points. Type IIA elliptic models have Type IIB descriptions in terms of branes and orientifolds in singular backgrounds. We showed that a necessary condition for there to exist a supergravity description of a theory, namely the equivalence of the Weyl and Euler anomalies, is satisfied in the elliptic models except at special points along the manifold of fixed points at which there are additional massless degrees of freedom, in which case we could not reliably calculate the anomalies. The condition $c-a=0$ also imposes severe restrictions on the types of nonelliptic models that can have supergravity descriptions: Any non-elliptic theory which can be obtained from Type IIA brane configurations with vanishing net sixbrane charge and NS5 brane linking numbers does not satisfy the supergravity condition $c-a=0$.

\section{Acknowledgments}

We are happy to thank Bo Feng, Martin Gremm, Ken Intriligator, Angel Uranga and Cumrun Vafa for useful discussions. This research is supported in part by the U.S. Department of Energy under cooperative agreement \#DE-FC02-94ER40818.

\section{References}


[1] R.G. Leigh and M.J. Strassler, "Exactly marginal operators and duality in fourdimensional N=1 supersymmetric gauge theory," Nucl. Phys. B447, 95 (1995) hepth/9503121.

[2] A. Karch, D. Lust and A. Miemiec, "New N=1 superconformal field theories and their supergravity description," hep-th/9901041.

[3] I.R. Klebanov and E. Witten, "Superconformal field theory on three-branes at a CalabiYau singularity," Nucl. Phys. B536, 199 (1998) hep-th/9807080.

[4] A.M. Uranga, "Brane configurations for branes at conifolds," hep-th/9811004.

[5] K. Dasgupta and S. Mukhi, "Brane constructions, conifolds and M theory," hepth/9811139.

[6] J. Park and A.M. Uranga, "A Note on superconformal N=2 theories and orientifolds," hep-th/9808161.

[7] S.S. Gubser, "Einstein manifolds and conformal field theories," Phys. Rev. D59, 025006 (1999) hep-th/9807164.

[8] V.A. Novikov, M.A. Shifman, A.I. Vainshtein and V.I. Zakharov, "Instantons And exact Gell-Mann-Low function of supersymmetric O(3) sigma model," Phys. Lett. 139B, 389 (1984).

[9] M. Henningson and K. Skenderis, "The Holographic Weyl anomaly," JHEP 07, 023 (1998) hep-th/9806087.

[10] J. Maldacena, "The Large N limit of superconformal field theories and supergravity," Adv. Theor. Math. Phys. 2, 231 (1997) hep-th/9711200.

[11] A. Giveon and D. Kutasov, "Brane dynamics and gauge theory," hep-th/9802067.

[12] A. Hanany and E. Witten, "Type IIB superstrings, BPS monopoles, and threedimensional gauge dynamics," Nucl. Phys. B492, 152 (1997) hep-th/9611230.

[13] E. Witten, "Solutions of four-dimensional field theories via M theory," Nucl. Phys. B500, 3 (1997) hep-th/9703166.

[14] M.R. Douglas and G. Moore, "D-branes, quivers, and ALE instantons," hep-th/9603167. 
[15] M.R. Douglas and B.R. Greene, "Metrics on D-brane orbifolds," Adv. Theor. Math. Phys. 1, 184 (1998) hep-th/9707214.

[16] A. Hanany and Y. He, "NonAbelian finite gauge theories," hep-th/9811183.

[17] B.R. Greene, C.I. Lazaroiu and M. Raugas, "D-branes on nonAbelian threefold quotient singularities," hep-th/9811201.

[18] R. von Unge, "Branes at generalized conifolds and toric geometry," hep-th/9901091.

[19] S. Gubser, N. Nekrasov and S. Shatashvili, "Generalized conifolds and 4-Dimensional N=1 SuperConformal Field Theory," hep-th/9811230.

[20] V.I. Arnold, S.M. Gusein-Zade, A.N. Varchenko, Singularities of differentiable maps, v.I,II, Boston: Birkhauser, 1988.

[21] E. Lopez, "A Family of $\mathrm{N}=1 \mathrm{SU}(\mathrm{N})^{* *} \mathrm{k}$ theories from branes at singularities," hepth/9812025.

[22] K. Landsteiner, E. Lopez and D.A. Lowe, "N=2 supersymmetric gauge theories, branes and orientifolds," Nucl. Phys. B507, 197 (1997) hep-th/9705199.

[23] C. Csaki, M. Schmaltz, W. Skiba and J. Terning, "Gauge theories with tensors from branes and orientifolds," Phys. Rev. D57, 7546 (1998) hep-th/9801207.

[24] I. Brunner, A. Hanany, A. Karch and D. Lust, "Brane dynamics and chiral nonchiral transitions," Nucl. Phys. B528, 197 (1998) hep-th/9801017.

[25] Z. Kakushadze, "Large N gauge theories from orientifolds with NS-NS B flux," hepth/9808048.

[26] N. Evans, C.V. Johnson and A.D. Shapere, "Orientifolds, branes, and duality of 4-D gauge theories," Nucl. Phys. B505, 251 (1997) hep-th/9703210.

[27] I. Brunner and A. Karch, "Branes at orbifolds versus Hanany Witten in six-dimensions," JHEP 03, 003 (1998) hep-th/9712143.

[28] J.D. Blum and K. Intriligator, "Consistency conditions for branes at orbifold singularities," Nucl. Phys. B506, 223 (1997) hep-th/9705030.

[29] A. Hanany and A. Zaffaroni, "Branes and six-dimensional supersymmetric theories," Nucl. Phys. B529, 180 (1997) hep-th/9712145. 
[30] S. Elitzur, A. Giveon, D. Kutasov and D. Tsabar, "Branes, orientifolds and chiral gauge theories," Nucl. Phys. B524, 251 (1998) hep-th/9801020.

[31] K. Landsteiner, E. Lopez and D.A. Lowe, "Duality of chiral N=1 supersymmetric gauge theories via branes," JHEP 02, 007 (1998) hep-th/9801002.

[32] A.M. Uranga, "Towards mass deformed $\mathrm{N}=4 \mathrm{SO}(\mathrm{n})$ and $\mathrm{Sp}(\mathrm{k})$ gauge theories from brane configurations," Nucl. Phys. B526, 241 (1998) hep-th/9803054.

[33] A. Sen, "F theory and orientifolds," Nucl. Phys. B475, 562 (1996) hep-th/9605150.

[34] E. Witten, "Anti-de Sitter space and holography," Adv. Theor. Math. Phys. 2, 253 (1998) hep-th/9802150.

[35] S.S. Gubser, I.R. Klebanov and A.M. Polyakov, "Gauge theory correlators from noncritical string theory," Phys. Lett. B428, 105 (1998) hep-th/9802109.

[36] D. Anselmi, D.Z. Freedman, M.T. Grisaru and A.A. Johansen, "Nonperturbative formulas for central functions of supersymmetric gauge theories," Nucl. Phys. B526, 543 (1997) hep-th/9708042.

[37] D. Anselmi, J. Erlich, D.Z. Freedman and A.A. Johansen, "Positivity constraints on anomalies in supersymmetric gauge theories," Phys. Rev. D57, 7570 (1998) hepth/9711035.

[38] A. Hanany, M.J. Strassler and A.M. Uranga, "Finite theories and marginal operators on the brane," JHEP 06, 011 (1998) hep-th/9803086.

[39] S. Kachru and E. Silverstein, "4-D conformal theories and strings on orbifolds," Phys. Rev. Lett. 80, 4855 (1998) hep-th/9802183.

[40] A. Hanany and A.M. Uranga, "Brane boxes and branes on singularities," JHEP 05, 013 (1998) hep-th/9805139.

[41] L.E. Ibanez, R. Rabadan and A.M. Uranga, "Anomalous U(1)'s in type I and type IIB $\mathrm{D}=4, \mathrm{~N}=1$ string vacua," hep-th/9808139. 